\title{
The Functions of BET Proteins in Gene Transcription of Biology and Diseases
}

\author{
Ka Lung Cheung*, Claudia Kim and Ming-Ming Zhou* \\ Department of Pharmacological Sciences, Icahn School of Medicine at Mount Sinai, New York, NY, United States
}

The BET (bromodomain and extra-terminal domain) family proteins, consisting of BRD2, $\mathrm{BRD} 3, \mathrm{BRD} 4$, and testis-specific BRDT, are widely acknowledged as major transcriptional regulators in biology. They are characterized by two tandem bromodomains (BDs) that bind to lysine-acetylated histones and transcription factors, recruit transcription factors and coactivators to target gene sites, and activate RNA polymerase II machinery for transcriptional elongation. Pharmacological inhibition of BET proteins with BD inhibitors has been shown as a promising therapeutic strategy for the treatment of many human diseases including cancer and inflammatory disorders. The recent advances in bromodomain protein biology have further uncovered the complex and versatile functions of BET proteins in the regulation of gene expression in chromatin. In this review article, we highlight our current understanding of BET proteins' functions in mediating protein-protein interactions required for chromatin-templated gene transcription and splicing, chromatin remodeling, DNA replication, and DNA damage repair. We further discuss context-dependent activator $v s$. repressor functions of individual BET proteins, isoforms, and bromodomains that may be harnessed for future development of BET bromodomain inhibitors as emerging epigenetic therapies for cancer and inflammatory disorders.

Keywords: bromodomain, bromodomain and extra-terminal domain proteins, gene transcription, inhibitors, cancer

\section{INTRODUCTION}

Bromodomain and extra-terminal domain (BET) family proteins, consisting of BRD2, BRD3, BRD4, and BRDT, are important transcription regulators, characterized by two N-terminal acetyl-lysine (Kac) binding bromodomains (BD1 and BD2) followed by an ET domain (Wu and Chiang, 2007; Zaware and Zhou, 2019) (Figure 1). BRD4 exists in three isoforms BRD4L, BRD4Sa, and BRD4Sb that reportedly have distinct functions in gene transcription (Wu et al., 2020). The C-terminal motif (CTM) is unique in BRD4L and not present in BRD4S and other BET proteins. Besides BD1, BD2, and CTM, BET proteins also have several functional motifs including a cluster of casein kinase 2 (CK2) phosphorylation sites (NPS and CPS) and a basic residue-enriched interaction domain (BID) (ChiangPhospho-BRD4, 2016). As arguably the most well-characterized domains in BET proteins, $\mathrm{BD} 1$ and $\mathrm{BD} 2$ domains, composed of the evolutionarily conserved four-helical bundle structure, function to recognize acetylated lysine in histones or transcription factors and act as readers of acetylation signals in gene transcription in chromatin (Dhalluin et al., 1999). The ET domain confers transcriptional activation by recruiting transcription regulatory proteins (Rahman et al., 2011; Zhang et al., 2016). Although the BET family proteins share similar functional domains (BD1, BD2, and ET), their expression patterns in tissues are distinct (Wang et al., 2021a). BRD2 and BRD3 are found to be expressed in the pancreas, testis, ovaries, brain, liver, spleen, lung, and kidney (Shang et al., 


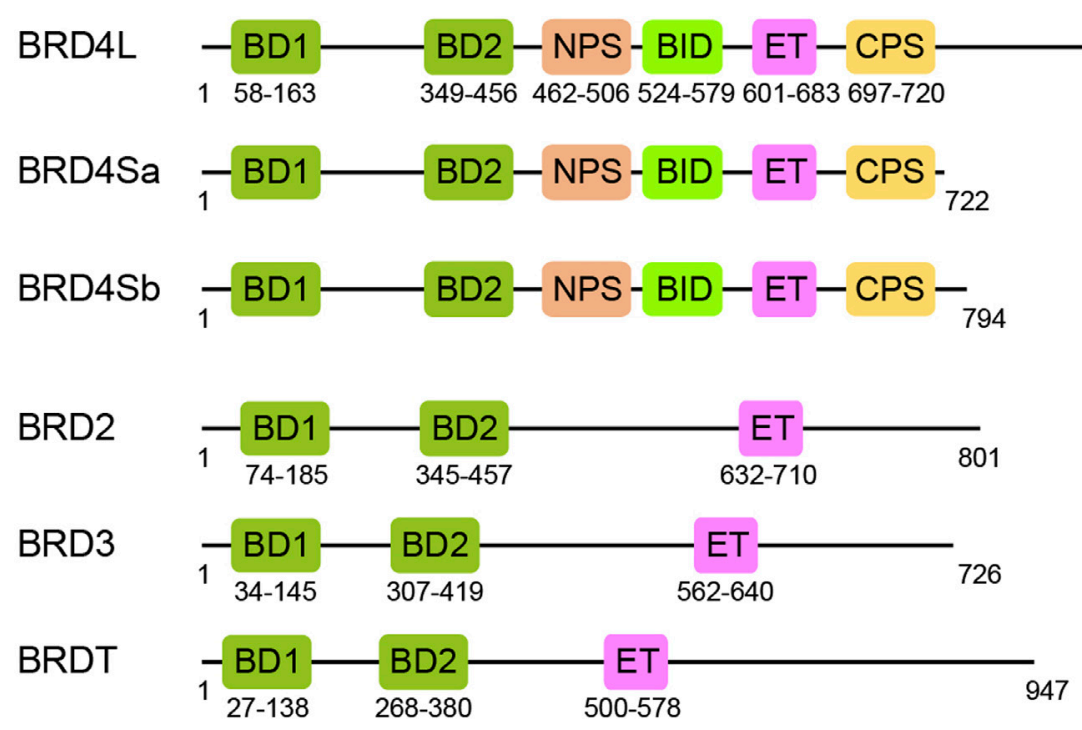

FIGURE 1 | Functional domains of BET family proteins. Schematic representation of domain architectures for BET proteins, BRD4 long and short isoforms, BRD2, BRD3, and BRDT: BD1, first bromodomain; BD2, second bromodomain; NPS, N-terminal phosphorylation site; BID, basic residue-enriched interaction domain; ET, extra-terminal; CPS, C-terminal phosphorylation site; CTM, C-terminal motif.

2009; Wang et al., 2009; Wang et al., 2021a). BRD4 is ubiquitously expressed, as it is functionally expressed in the bone marrow and lymphoid compartment in addition to other tissues, while BRDT is selectively expressed in the testis and ovaries (Wang et al., 2021a). In cells, BET proteins also display non-redundant functions, which are often cooperative in activation of gene transcription (Cheung et al., 2017a). Therefore, it is important that we gain a deeper understanding of the overlapping and distinct functions of BET proteins in gene transcription and diseases.

The most well-studied function of BET proteins, particularly BRD4, is the role in transcriptional elongation (Hargreaves et al., 2009). In a simplistic model of transcriptional activation, RNA polymerase (Pol)-II recruitment to regulatory sites such as enhancers and promoters is the first limiting step to activate gene transcription. This simple model has been challenged and refined by the concept of transcriptional elongation, which accounts for the fast kinetics of gene activation and transcriptional memory upon stimuli. In a seminal study by Medzhitov and colleagues using bone marrow-derived macrophages to address the difference in transcriptional kinetics of primary response genes (PRGs) and secondary response genes (SRGs), it was found that PRGs, but not SRGs, have pre-assembled RNA Pol-II at their promoters in unstimulated conditions. Upon stimulation, signal-dependent recruitment of $\mathrm{BRD} 4 / \mathrm{p}-\mathrm{TEFb}$ to acetylated histones of these sites facilitates RNA Pol-II transcriptional elongation and mRNA processing (Hargreaves et al., 2009). In addition to direct effect on promoter sites, BRD4 also exerts pronounced effect on anti-pause enhancers (A-PEs) that activates promoterbound RNA Pol-II through a mechanism termed "transcriptional pause-release" (Anand et al., 2013; Liu et al., 2013). BRD4 recruits demethylase JMJD6 on A-PEs to erase
H4R3me2s, which is read by 7SK snRNA, and demethylation/ deactivation of 7SK small nuclear RNA (snRNA), to inhibit the binding and function of the 7SK snRNA/HEXIM repressive complex, therefore allowing for pause-release of RNA Pol-II (Liu et al., 2013). In addition to their role in gene transcriptional elongation, recent technological advancements have also led to the discovery of other functions of BET proteins, including chromatin organization, super-enhancer assembly, and condensate formation. In this review article, we first discuss in detail various molecular mechanisms that modulate BET protein expression and activity in human diseases, which are not well-appreciated and lack systematic review. We then summarize the recent advances in our understanding of the multi-faceted functions of BET proteins in gene transcription in chromatin. Finally, we highlight the more context-dependent distinct functions of BET proteins including different isoform and repressor functions, providing rationales and opportunities for more selective and potentially less toxic therapeutic strategies targeting BET proteins.

\section{MOLECULAR MECHANISMS MODULATING BROMODOMAIN AND EXTRA-TERMINAL DOMAIN PROTEIN EXPRESSION AND FUNCTION}

\section{Mechanisms Regulating Expression of Bromodomain and Extra-Terminal Domain Proteins}

In cancer, the expression of BET proteins is often upregulated to enhance oncogenic activities. Studies are beginning to reveal exactly how the BET proteins are aberrantly overexpressed in 


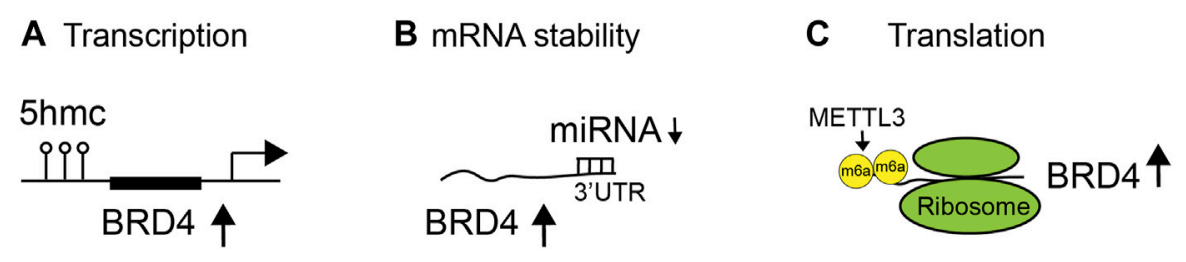

FIGURE 2 | Regulation of BET protein expression and functions in diseases. Expression and activity of BET proteins are regulated at different levels, including (A) transcription by 5-hydroxymethylcytosine (5-hmC) at the BRD4 promoter, (B) messenger RNA (mRNA) stability by binding of microRNA (miRNA) to the $3^{\prime}$ untranslated region (UTR) of BRD4 mRNA transcripts, and (C) translation by $N^{6}$-methyladenosine $\left(m^{6} A\right)$ modification of mRNA (Rahman et al., 2011$)$.

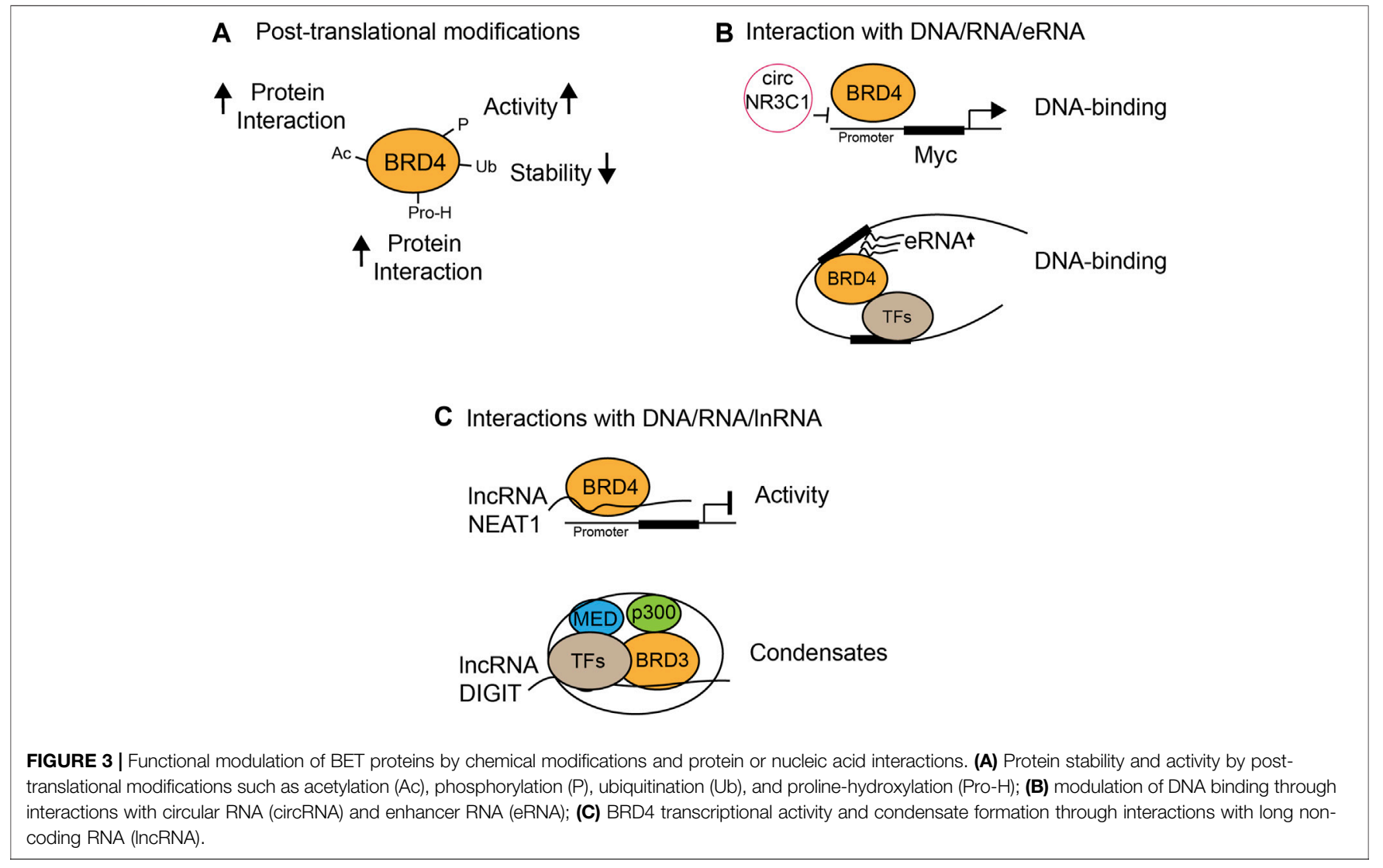

disease contexts. For instance, it has been demonstrated that 5hydroxymethylcytosine $(5-\mathrm{hmC})$ can regulate $\mathrm{BRD} 4$ transcript expression through 5 -hmC enrichment at open chromatin and BRD4 promoter sites, correlating with upregulated transcriptional expression of BRD4 in pancreatic neoplasia (Bhattacharyya et al., 2017) (Figure 2A). Additionally, miRNA can bind to the $3^{\prime}$ untranslated region (UTR) of BRD4 to regulate the mRNA abundance or stability of BRD4 in diseases (Figure 2B). miR-29b targets the $3^{\prime}$-UTR of BRD4 to regulate the expression of BRD4 and has an inverse correlation (low miR$29 \mathrm{~b}$ and high BRD4) in chronic obstructive pulmonary disease (COPD) patients (Tang et al., 2019). miR-1340 targets BRD3 and BRD4 to suppress tumor cell growth (Tonouchi et al., 2018). Diminished miR-29b level leads to enhanced BRD4 level and activation in cutaneous T-cell lymphoma (Kohnken et al., 2018). miR-9 suppresses BRD4 expression in cardiomyocytes. In response to pathological stress stimuli, miR-9 is downregulated, leading to de-repression of BRD4 and enrichment of BRD4 on super-enhancers (SEs) associated with pathological cardiac genes (Stratton et al., 2016). Notably, long non-coding RNA (lncRNA) LINC00346, in contrast, acts as a sponge for miR-188-3p and blocks the repression of BRD4 by miR-188-3p in pancreatic cancer (Shi et al., 2019). Finally, upon knockdown of METTL3, endogenous BRD4 expression is strongly reduced, independently of mRNA abundance, leading to the discovery that METTL3-eIF3h mediates $N^{6}$ methyladenosine ( $\left.\mathrm{m}^{6} \mathrm{~A}\right)$ modification (Rahman et al., 2011) and circularization of BRD4 mRNA to enhance translation 
and promote oncogenesis (Choe et al., 2018) (Figure 2C). Collectively, these studies illustrate that BRD4 overexpression in cancer is likely due to enhanced mRNA transcription, abundance, and/or translation.

\section{MODULATION OF BROMODOMAIN AND EXTRA-TERMINAL DOMAIN PROTEIN FUNCTIONS BY POST-TRANSLATIONAL MODIFICATIONS}

Post-translational modifications of BET proteins, including acetylation, phosphorylation, ubiquitination, and proline hydroxylation, regulate protein stability and functions (Figure 3A). Acetylation of BRD4 enhances its interactions with other proteins. PCAF acetylates transcription factor ISX at Lys69 and BRD4 at Lys332, which form a complex and translocate into the nucleus to promote transcriptional expression of genes important for epithelial-mesenchymal transition and metastasis (Wang et al., 2020). Phosphorylation of BRD4 (pBRD4) is often associated with enhanced BRD4 oncogenic activities in cancer, as demonstrated by hyperphosphorylated BRD4 in NUT midline carcinoma, potentially mediated by CDK9 (Wang et al., 2017). In addition, kinase (CK2) and phosphatase (PP2A) that phosphorylate and dephosphorylate BRD4, respectively, to modulate its function in chromatin localization, transcription factor recruitment, and cancer progression have been reported (ChiangPhospho-BRD4, 2016). Importantly, CK2-mediated phosphorylation of a conserved acidic region in BRD4 dictates whether this region contacts with juxtaposed bromodomain (BD2) or an adjacent basic region, therefore determining if $\mathrm{BD} 2$ is free to bind acetylated-TF p53 (Wu et al., 2013). The same mechanism also applies in cocaine-seeking behavior and memory function in mice, as cocaine or neuronal stimulation induces the activation and binding of pBRD4 to the key gene promoter, which can be attenuated by CK2 blockade (Korb et al., 2015; Guo, 2020). Therefore, apart from modulating BRD4 bromodomains' interactions with chromatin, strategies targeting activated pBRD4 may represent an alternative and promising approach.

Equally important as acetylation and phosphorylation is BRD4 ubiquitination that has been linked to aberrant degradation of BRD4 leading to BET-BD inhibitor resistance. The most wellcharacterized example of BRD4 ubiquitination is controlled by the SPOP-DUB3 switch. De-ubiquitinase DUB3 binds to BRD4, but not $\mathrm{BRD} 2 / 3$, via a specific C-terminal motif (CTM), antagonizes SPOP-mediated BRD4 ubiquitination, and promotes BRD4 de-ubiquitination and stabilization, leading to BET-BD inhibitor resistance in cancer cells (Jin et al., 2018). In prostate cancer, specific SPOP mutation can also confer resistance to BET-BD inhibitors through stabilization of BRD4 (Dai et al., 2017) and enhanced Akt-mTORC1 activation as a result of BRD4 stabilization (Zhang et al., 2017). Interestingly, SPOP mutants in different types of cancer yield opposing effects on BET protein degradation by differential ubiquitination and sensitivity to BET-BD inhibitors. Endometrial cancer-associated
SPOP mutations preferentially degrade BET proteins, while prostate-cancer-specific SPOP mutation results in impaired degradation of BET proteins, as mutations of SPOP degrons in BRD4 determine whether they bind BET proteins or not, thus influencing BET ubiquitination (Janouskova et al., 2017). Finally, the less well-characterized post-translational modifications of BRD4 include proline hydroxylation and isomerization. Prolyl hydroxylase domain protein PHD2 is the key regulatory enzyme of BRD4 proline hydroxylation (P536) that significantly influences BRD4 interaction with CDK9, CDK1, and MCM5 and affects BRD4 transcriptional activation, but not BRD4 degradation or abundance (Erber et al., 2019). Prolyl isomerase PIN1 regulates the stability and transcriptional activity and oncogenic potential of BRD4 in gastric cancer cells. PIN1 recognizes BRD4 Thr204 and enhances BRD4 stability by inhibition of ubiquitination. PIN1 also catalyzes the isomerization of BRD4 Pro205 and induces its conformation change to promote CDK9 interaction and transcriptional activity and gastric cancer cell proliferation ( $\mathrm{Hu}$ et al., 2017).

\section{RNA/DNA-MEDIATED MECHANISMS MODULATING FUNCTIONS OF BROMODOMAIN AND EXTRA-TERMINAL DOMAIN PROTEINS}

It has been increasingly recognized that interactions of BET proteins with RNA/DNA determine the functional outcomes of BET proteins in gene transcription. For example, RNA has been shown to regulate the DNA-binding activity or transcriptional activity of BRD4 in different contexts. Circular RNA circNR3C1 dissociates BRD4 from binding to the $\mathrm{c}-\mathrm{Myc}$ promoter in bladder cancer cell lines and suppresses bladder cancer progression as an endogenous blocker of BRD4 (Xie et al., 2020) (Figure 3B). On the contrary, lncRNA could inhibit BRD4 transcriptional activity. BRD4 binds several long non-coding RNAs, and one of them is IncRNA NEAT1 that interacts with BRD4/ WDR 5 and forms a low-activity complex. BET-BD inhibitors dissociate NEAT1 from BRD4/WDR5 and restore the acetyltransferase activity of BRD4 and promote WDR5dependent histone trimethylation, leading to transcriptional activation (Pistoni, 2021) (Figure 3C). Interestingly, BRD3 forms phase-separated condensates, of which formation is promoted by lncRNA DIGIT. BRD3-DIGIT co-occupies enhancer sites with histone $\mathrm{H} 3 \mathrm{~K} 18 \mathrm{ac}$ to cooperatively form protein-lncRNA phaseseparated condensates and has a broad role in gene transcription (Daneshvar et al., 2020). Notably, enhancer RNA (eRNA) is another type of RNA that interacts with BET family proteins to promote enhanced chromatin engagement and transcriptional activation. While all BET proteins interact with eRNA through their bromodomains, BRD2, BRD3, and BRD4 interaction with eRNAs is much stronger as compared to BRDT. Specifically, BRD4-BDs 


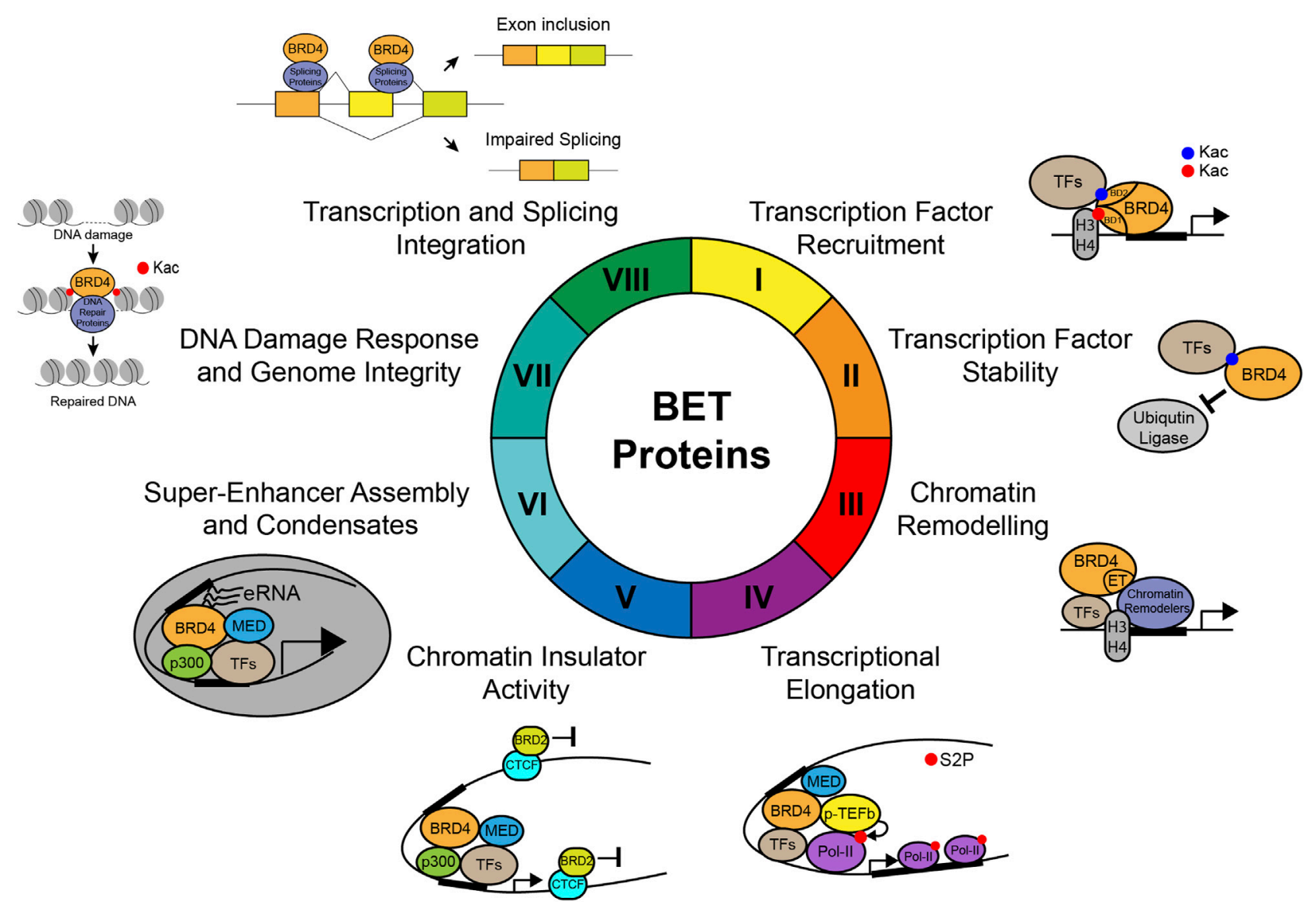

FIGURE 4 | Molecular functions of BET proteins in gene transcription in chromatin. Illustration of the molecular functions of BET proteins in regulation of gene transcription in chromatin, including (I) transcription factor recruitment, (II) transcription factor stability, (III) chromatin remodeling, (IV) transcriptional elongation through phosphorylation of RNA Pol-II Ser2, (V) chromatin insulator activity, (VI) super-enhancer assembly and condensates, (VII) DNA damage response and genome integrity, and (VIII) integration of gene transcription and splicing.

function cooperatively as a docking site for direct binding of eRNA to increase BRD4 binding to acetylated histones in vitro, BRD4 enhancer recruitment, and transcriptional coactivator activities (Rahnamoun et al., 2018). eRNA production is a key feature of enhancer activation and is BRD4-dependent as the BET-BD inhibitor JQ1 reduces eRNA abundance on the enhancer, suggesting BRD4 controls a feedback loop that enhances eRNA production on the enhancer, which in turn promotes BRD4 chromatin engagement and transcriptional activation. Finally, it has been shown that interactions of BET-BDs with DNA facilitate acetylation-dependent bivalent nucleosome recognition by BRDT (Miller et al., 2016). BRDT interacts with nucleosomes through its BD1 but not BD2, and acetylhistone recognition is complemented by a bromodomain-DNA interaction, as simultaneous recognition enhances nucleosome binding affinity and specificity. Conservation of DNA binding in BDs of BET therefore indicates the bivalent nucleosome recognition as a key feature (Miller et al., 2016). Collectively, these results highlight the emerging concept that RNA/DNA-mediated mechanisms play a crucial role in the control of BRD4 chromatin binding and activity that contributes to gene transcriptional controls in diseases.

\section{MULTI-FACETED FUNCTIONS OF BROMODOMAIN AND EXTRA-TERMINAL DOMAIN PROTEINS IN GENE TRANSCRIPTION IN CHROMATIN}

\section{Histone/Transcription Factor Recruitment of Bromodomain and Extra-Terminal Domain Proteins Through the Bromodomains}

One of the major molecular functions of BET proteins is to act as an epigenetic reader to relay signals from lysine-acetylated transcriptional factors or histones to regulate gene transcription in chromatin (Figure 4). Intriguingly, recent studies also suggested that histone lysine acyl-related modifications such as crotonylation and butyrylation may play 
a role in BD-mediated recognition (Soffers et al., 2016). While a subset of bromodomain-containing proteins such as BRD9 and TAF1 recognize histone crotonylation and butyrylation, their binding affinities with crotonylated/butyrylated peptides are much weaker than those with acetylated peptides (Flynn et al., 2015). On the contrary, the YEATS domain of AF9 has been shown to strongly bind histone crotonylation compared to acetylation (Li et al., 2016). The BET-BD recognition of acetylation marks instead of other acylation marks is further strengthened by the discovery that histone butyrylation competes to prevent, instead of facilitating, BRDT binding to H4K5 (Goudarzi et al., 2016). Therefore, these studies argue that BDs of BET proteins likely function as a specific reader for lysine acetylation, and not other types of lysine acylation.

Studies have identified different mechanisms as to how BET proteins are recruited to regulatory sites of key genes. Acetylated transcription factors (TFs) and histones bind, recruit, and work with BRD4 to drive super-enhancer formation and gene expression. In acute myeloid leukemia (AML), acetylated histone $\mathrm{H} 4$ and hematopoietic transcription factors interact with BRD4 via a common $\mathrm{K}^{\mathrm{ac}} \mathrm{GGK}^{\mathrm{ac}}$ motif to recruit BRD4 to regulatory sites (Roe et al., 2015). Similarly, the second bromodomain (BD2) of BRD4 interacts with diacetylated Twist via the same motif to promote tumorigenesis in basallike breast cancer (Shi et al., 2014). The functional transcription factor-BET binding is characterized by conserved binding mechanisms, as each BET-BD1/2 can recognize, in a single binding pocket, a dual-Kac motif of $\mathrm{K}^{\mathrm{ac}} \mathrm{XXK}^{\mathrm{ac}}$ present in transcriptional factors including TWIST, GATA1, E2F1, and MyoD1 (Patel et al., 2021a). Crystal structures of BD1 of BRDT and BRD2 bound to a diacetylated histone $\mathrm{H} 4$ peptide show that two acetylation marks are cooperatively recognized by one binding pocket, and this mode of ligand recognition applies to other bromodomain-containing proteins as well (Nakamura et al., 2007; Morinière et al., 2009). Histone variants are another important regulatory control of aberrant gene transcription in human diseases including cancer. H2A.Z, one of the most wellstudied variants, was shown to be co-purified with BRD2. The NMR study has revealed that lysine acetylation on the N-terminal tail of H2A.Z (K4acK7ac and K7acK11ac) interacts with BDs of $\mathrm{BRD} 2, \mathrm{BRD} 3$, and BRD4 and is reminiscent of the characteristic binding of the N-terminal tail of H4-K5acK8ac to BRD4-BD1. Thus, histone variant-BET protein binding also follows the common feature that a single bromodomain preferentially binds diacetylated motifs commonly in a $\mathrm{K}^{\mathrm{ac}} \mathrm{XXK}^{\mathrm{ac}}$ motif (Patel et al., 2021b). Finally, a systematic proteomics study confirmed that BET-BDs recognize $\mathrm{K}^{\mathrm{ac}} \mathrm{XXK}^{\mathrm{ac}}$ motifs on histones where $\mathrm{XX}$ can be GG, GS, DG, AA, AP, AV, AQ, AR, SA, VL, LN, TA, and TP (Lambert et al., 2019). Interestingly, "histone-like" XX sequences were found in the human proteome, recognized by $\mathrm{BD} 1$ and/or $\mathrm{BD} 2$ domains, and a diverse flanking sequence can drive binding to either bromodomain (Lambert et al., 2019). These studies highlighted the binding specificity and versatility of BET-BD1/2 domains and $\mathrm{K}^{\mathrm{ac}} \mathrm{GGK}^{\mathrm{ac}}$ motif in both histones and non-histone proteins.

Apart from the simple paradigm that $\mathrm{H} 4 \mathrm{~K} 5 / 8 / 12$ acetylation mediates BRD4 binding, more complex histone crosstalk and combinatorial histone modifications create a platform for BRD4 binding. For example, serum stimulation of cells induced PIM1 kinase to phosphorylate the pre-acetylated histone $\mathrm{H} 3(\mathrm{H} 3 \mathrm{~K} 9 \mathrm{ac} /$ S10ph) at the FOSL1 enhancer. 14-3-3 then acts as an adaptor protein that binds the phosphorylated histone to further recruit the histone acetyltransferase MOF and trigger the acetylation of H4K16ac for BRD4 binding (Zippo et al., 2009). By screening over 30 representative $\mathrm{BD}$ proteins against histone peptide arrays with systematic changes in histone combination marks, it was confirmed that BRD4 recognizes a combination of marks such as acetylation and phosphorylation rather than singly acetylated sequences (Filippakopoulos et al., 2012). Thus, BRD4 binding can be a result of a cascade of events leading to a nucleosomal platform that recruits BRD4.

\section{BROMODOMAIN AND EXTRA-TERMINAL DOMAIN PROTEINS REGULATE STABILITY OF TRANSCRIPTION FACTORS}

BRD4 interaction with transcription factors can enhance binding partner stability or induce degradation through different mechanisms (Figure 4). In castration-resistant prostate cancer (CPRC), the histone demethylase JMJD1A was shown to regulate activities of androgen receptor (AR) and c-Myc. JMJD1A protein stability is controlled by ubiquitin ligase STUB1. Histone acetyltransferase (HAT) p300 acetylates JMJD1A at K421 that recruits BRD4 to block JMJD1A from degradation, which is reversed by p300 HAT or BET-BD inhibitors (Xu et al., 2020). Similarly, BRD4 C-terminal domain (CTD) interacts with HPV16 (human papillomavirus) E2 protein, which blocks E2 interaction with cullin-3, leading to reduced E2 ubiquitination and enhanced E2 stability (Zheng et al., 2009). Finally, BRD4 recognizes K146ac and K187ac on Snail to prevent Snail from interacting with E3 ubiquitin ligase FBX14 and b-Trcp1, thereby inhibiting Snail polyubiquitination and proteasomal degradation, revealing a non-canonical post-transcriptional regulatory function of BRD4 in maintaining cancer cell growth and metastasis (Qin et al., 2019). Conversely, BRD4 was also shown to induce degradation by phosphorylation of TFs. It has been reported that oncogenic protein MYC stability is controlled by phosphorylation, as pThr58 signals degradation, while pSer62 leads to stabilization. BRD4, with its reportedly intrinsic kinase and histone acetyltransferase activity, phosphorylates MYC at Thr58, leading to MYC ubiquitination and degradation (Devaiah et al., 2020). These studies suggest one major mechanism of BET proteins in acetylation-dependent stabilization of interacting TFs, which likely competes off the binding of E3 ubiquitin ligases.

\section{BROMODOMAIN AND EXTRA-TERMINAL DOMAIN PROTEIN INTERACTIONS WITH CHROMATIN REMODELERS THROUGH THE ET DOMAIN}

The ET domain of BET proteins has been shown to be involved in protein-protein interactions in gene transcription (Figure 4). As 
shown by a proteomic analysis, the ET domain of BRD4 interacts with multiple chromatin remodeling proteins including NSD3, JMJD6, CHD4, and GLTSCR1 (Rahman et al., 2011). As demonstrated by further detailed structural and functional characterization (Liu et al., 2013; French et al., 2014; Shen et al., 2015; Zhang et al., 2016; Konuma, 2017; Gatchalian, 2018; Wong, 2019; Lam, 2020; Lee, 2020), the ET domain recognizes a short peptide motif that is conserved across chromatin remodelers and transcriptional regulators. This is supported by a study showing that BRD3 binds to an array of chromatin remodeling complexes, including the $\mathrm{NuRD}, \mathrm{BAF}$, and INO80 complexes, via a short linear "KIKL" motif in one of the complex subunits (Wai et al., 2018). In addition, the ET domain-binding motif, which shares similar amino acid sequences from other BRD4-ET-binding proteins such as CHD4 and NSD2/3, also mediates interaction of BRD4-ET with ASXL3 and functionally links BRD4 to the BAP1 complex in a small-cell lung cancer (SCLC) subtype (Szczepanski, 2020). Interestingly, the detailed NMR structural analysis of JMJD6/BRD4-ET domain complex by Zhou and colleagues revealed that a JMJD6 peptide (Lys84-Asn96) adapts an $\alpha$-helix when bound to the conserved hydrophobic core of the ET domain (Konuma, 2017). This ligand recognition mode is different from the ET domain recognition of NSD3, LANA of herpesvirus, and integrase of MLV that proceeds with the formation of a small two-strand $\beta$-sheet (Zhang et al., 2016; Konuma, 2017). Collectively, these studies provided the structural and molecular basis on how the BRD4-ET domain recognizes a conserved BRD4-binding motif on chromatin remodeling complex proteins to form a functional complex for gene transcription.

\section{BROMODOMAIN AND EXTRA-TERMINAL DOMAIN PROTEINS FACILITATE RNA POL-II PAUSE-RELEASE AND TRANSCRIPTIONAL ELONGATION}

BET proteins are known to facilitate RNA Pol-II release from promoter-proximal pause regions through recruitment and activation of p-TEFb (CDK9/cyclin T1) that triggers transcriptional elongation (Yang et al., 2005; Jang et al., 2005) (Figure 4). BRD4 association with $\mathrm{p}$ - $\mathrm{TEFb}$ is required to form an active complex for stimulation of RNA Pol-II elongation on both coding and non-coding enhancer RNAs (Kanno et al., 2014), and the functional importance has been demonstrated in different cellular contexts including embryonic stem cells (ESCs) (Di Micco et al., 2014), heart cells (Anand et al., 2013), and macrophages (Hargreaves et al., 2009). Mechanistically, BRD4BD2 interactions with tri-acetylated cyclin T1 (K380ac, K386ac, K390ac) and cyclin T1 with BRD4-PID (C-terminal $\mathrm{p}$ - $\mathrm{TEFb}$-interacting domain) are required for the release of $\mathrm{p}-\mathrm{TEFb}$ from an inactive HEXIM1-7SK small nuclear ribonucleoprotein complex and for full transcriptional activation of p-TEFb (Schröder et al., 2012). In addition to $\mathrm{BRD} 4, \mathrm{p}-\mathrm{TEFb}$ can also be recruited to target genes by a super-elongation complex (SEC) in the development of cardiomyocyte hypertrophy following activation of distinct GPCR $\left[\alpha_{1}\right.$-adrenergic receptor $\left(\alpha_{1}-A R\right)$ or endothelial receptor (ETR)]. However, while the SEC is required for both activation modes, BRD4 is selectively required for $\alpha_{1}$-AR response, suggesting the BRD4/p-TEFb complex can be differentially activated in response to distinct signaling pathways (Martin, 2020).

Importantly, BRD4 is involved in multiple steps of the transcriptional elongation hierarchy. Using an auxin-inducible degradation (AID) system to rapidly deplete targeted proteins, it was shown that CDK9-containing BRD4 is required for genomewide release of RNA Pol-II from promoter-proximal pausing under normal conditions, while BRD2 is required for global RNA Pol-II positioning at enhancers (Zheng et al., 2021). As aforementioned, in addition to coding genes, BRD4 mediates its function through enhance regions, as it assists enhancer RNA synthesis through transcriptional elongation. In addition, BRD4 was also shown to interact with JMJD6 (a histone arginine demethylase) to demethylate H4R3 at the target gene antipause enhancer to release RNA Pol-II from promoterproximal pause regions, leading to aberrant gene expression in glioblastoma (Wong, 2019). However, although BET and mediator proteins have been shown to form phase condensates and are crucial for super-enhancer function, dissolving phase condensates reduces BRD4 and mediator binding to enhancers but does not disrupt enhancer-promoter interactions (Crump, 2021). In addition, targeting cells with BET-BD inhibitors has a strong impact on transcription but very little impact on enhancer-promoter interactions (Crump, 2021). These findings suggest that activation of transcription and maintenance of enhancer-promoter interactions are separable events and that BRD4 acts in activation of gene transcription through $\mathrm{p}$-TEFb-mediated RNA Pol-II release from enhancer and promoter regions much more than in the maintenance of enhancer-promoter interactions.

\section{CHROMATIN STRUCTURE ORGANIZATION BY BROMODOMAIN AND EXTRA-TERMINAL DOMAIN PROTEINS}

BET proteins actively organize chromatin to define transcriptional and architectural boundaries for gene transcription (Figure 4). Chromatin architectural/insulator protein CTCF recruits BRD2, but not BRD4, and they colocalize genome-wide to insulate regulatory influence from unrelated genes, indicating that CTCF and BRD2 form a transcriptional boundary, of which activity is dependent on BRD2 (Hsu et al., 2017). This is further supported by preferential binding of BRD2 to $\mathrm{CTCF} /$ cohesin components, illustrating the distinct functions between BRD2 and BRD4 in that BRD2 are involved in boundary activities, while BRD4 promotes transcriptional elongation (Cheung et al., 2017a). Although BRD2's function in chromatin organization is evident, BRD4's ability to associate with acetylated chromatin is nonetheless also important for maintenance of higher order 
chromatin structures and defining transcriptional boundaries (Wang et al., 2012). It has been reported that BRD4 interacts and forms a complex with $\mathrm{p} 300 / \mathrm{CBP}$ through its BDs to enhance its C-terminal intrinsic HAT activity, which enhances p300 enzymatic activity to deposit H3K27 and H3K56 acetylation, regulates Brg1 binding to chromatin, and thus modifies the chromatin structure (Wu et al., 2018). In NUT (nuclear protein in testis) midline carcinoma (NMC), a highly aggressive subtype of squamous cell cancer, BRD4-NUT fusion oncoprotein enhances the interaction of p300 and BRD4, leading to a massive feed-forward expansion of transcriptionally inactive hyperacetylated chromatin domains (Reynoird et al., 2010). Finally, BET proteins also actively evict histones to facilitate gene transcription. For example, interferon (IFN) stimulation induces GCN5-mediated acetylation of H2A.Z, leading to $\mathrm{BRD} 2$ engagement, chromatin remodeling, and eviction of H2A.Z. This in turn enables ISGF3 to bind and activate interferon-stimulated gene (ISG) expression, providing an example of how BRD2 can evict nucleosomes to facilitate gene transcription (Au-Yeung and HorvathHistone, 2018). BRD4 has reported intrinsic HAT activity that acetylates histone H3K122, a residue critical for nucleosome stability, and evicts nucleosomes from chromatin, resulting in chromatin decompaction, thus linking the chromatin structure and gene transcription (Devaiah et al., 2016). Similarly, PHD finger protein 7 (PHF7) mediates BRDT stability (Kim et al., 2020), which is required for recognition of acetylated chromatin through the BD1 of BRDT (Dhar et al., 2012) and removal of histones to facilitate histone-toprotamine exchange, a critical step for sperm nuclear condensation during spermatogenesis (Kim et al., 2020). These studies together demonstrate the critical histone removal functions of BET proteins in different functional contexts.

\section{BROMODOMAIN AND EXTRA-TERMINAL DOMAIN PROTEINS IN SUPER-ENHANCER ASSEMBLY}

Pharmacological inhibition of the BET bromodomains has been regarded as a new therapeutic strategy targeting the transcriptional expression of $\mathrm{c}-\mathrm{Myc}-$ dependent and Mycdependent target genes. JQ1 is among the best known bromodomain inhibitors that effectively displaces BRD4 interaction from acetylated histones (Filippakopoulos et al., 2010). In vivo efficacy of JQ1 in cancer cell growth inhibition has been confirmed in mouse models of various cancers including squamous carcinoma (Filippakopoulos et al., 2010), multiple myeloma (Delmore et al., 2011), acute myeloid leukemia (AML) (Zuber et al., 2011), MLL-fusion leukemia (Dawson et al., 2011), castration-resistant prostate cancer (Asangani et al., 2014), breast cancer (Shu et al., 2016), melanoma (Fontanals-Cirera et al., 2017), and inflammation in activated macrophages (Nicodeme et al., 2010).

In a mechanistic study on how inhibition of the widely expressed transcriptional coactivator BRD4 leads to selective inhibition of the Myc oncogene in multiple myeloma, it was shown that BRD4 and mediator co-occupy in chromatin in a small set of exceptionally large super-enhancers associated with genes important for multiple myeloma. JQ1 led to preferential loss of BRD4 at super-enhancers and transcriptional elongation on those genes, including Myc (Lovén et al., 2013). Superenhancers are defined as a long class of clusters of regulatory regions bound by TFs that control cell identity and disease genes. Components of super-enhancers also facilitate recruitment of Drosha/DGCR8 for primary-microRNA (pri-miRNA) processing to boost cell-specific miRNA production. Therefore, BRD4 and super-enhancers facilitate transcription of key identity genes, including coding mRNA and biogenesis of master miRNAs that are crucial for cell identity (Suzuki et al., 2017). On the contrary, the testis-specific protein BRDT directs super-enhancer activity in a subset of esophageal squamous cell carcinoma (ESCC). BRDT is aberrantly expressed in over $30 \%$ of ESCC, enhances $\Delta \mathrm{Np} 63$ (a defining factor of the squamous subtype)dependent SE-associated genes, and controls the migratory potential of ESCC cells (Wang et al., 2021b). The concept of super-enhancer also led to more studies to identify key genes and reveal mechanisms of BET proteins in disease-related cell types and genes such as rheumatoid arthritis synovial fibroblasts (Krishna et al., 2021), ESC pluripotency genes (Di Micco et al., 2014), and senescence-associated secretory genes (Tasdemir et al., 2016).

\section{BROMODOMAIN AND EXTRA-TERMINAL DOMAIN PROTEINS IN CONDENSATE FORMATION}

Liquid-liquid phase separation, characterized by protein and other bimolecular condensates, has emerged as a crucial membrane-less compartment implicated in biological processes including chromatin reorganization, as well as in human diseases such as neurodegenerative disorders and cancer (Krainer, 2021) (Figure 4). One major function of BRD4 in organization and regulation of eukaryotic genomes is through cocondensate-mediated concentration of transcriptional apparatus. For example, phase separation of DNA-binding cofactor TEAD4, coactivators BRD4 and MED1, and elongation factor CDK9 locally concentrates transcriptional activators to facilitate the expression of TAZ-target genes and TAZ-mediated growth, development, and tumorigenesis ( $\mathrm{Lu}$ et al., 2020). A link between super-enhancers (SEs) and cocondensates was illustrated by findings that SE-enriched transcriptional coactivators BRD4 and MED1 form nuclear puncta at SEs that exhibit properties of liquid-like condensates to compartmentalize and concentrate transcription apparatus from nuclear extracts (Sabari, 2018). Intrinsically disordered regions (IDRs) of HAT transcription coactivator p300 interact with TF-transactivation domains (TADs), forming cocondensates that promote p300 transactivation and stabilize TF-p300 assembly, priming the recruitment of coactivator $\mathrm{BRD} 4$, and contribute to transcriptional bursting regulation and cooperative gene control (Ma, 2021). However, histone acetylation by p300 also antagonizes chromatin phase separation, while BRD4 helps highly acetylated chromatin to 
form a new phase-separated state with droplets of distinct physical properties (Gibson et al., 2019). Previous reports have shown that protein condensation is mainly driven by the action of electrostatic and hydrophobic interactions that underlies the phase separation of proteins at or below physiological ionic strength, although it was also recently shown that BRD4 can undergo phase separation at a high salt concentration driven by hydrophobic and non-ionic interactions, and is mechanistically different from the low-salt regime (Krainer, 2021). Collectively, these studies demonstrate the versatile functions of BRD4 in the regulation of co-condensate formation through active stabilization of the co-condensates formed between TFs, coactivators, p300, and acetylated histones to attain superenhancer functions in gene transcription.

\section{BROMODOMAIN AND EXTRA-TERMINAL DOMAIN PROTEINS IN COORDINATION OF GENE TRANSCRIPTION AND SPLICING}

It is becoming increasingly clear that transcriptional elongation is intricately coupled with splicing, transcriptional termination, and genome stability (Jonkers and Lis, 2015) (Figure 4). In castrationresistant prostate cancer (CRPC), BET-BD inhibition affects the regulation of alternative splicing of androgen receptor (AR), leading to reduced AR-V7 expression and abrogating AR signaling and growth of CRPC patient-derived models (Welti et al., 2018). In T-cell acute lymphoblastic leukemia (T-ALL) cancer cells and during thymocyte differentiation in vivo, distinct patterns of alternative splicing are associated with BRD4 deletion (Uppal et al., 2019). Specifically, BRD4 directly interacts with splicing machinery (FUS, HnRNPM, HnRNPL, U1-70, and U1A), suggesting interplay of BRD4 and splicing factors that modulates exon usage (Uppal et al., 2019). Finally, BRD4 also regulates splicing during heat shock, as BRD4 depletion leads to significant splicing inhibition (in particular intron retention) in heat-treated cells, leading to decreased mRNA abundancy of affected transcripts, which is mediated by heat shock factor 1 (HSF1)-BRD4 interaction to recruit BRD4 to nuclear stress bodies (Hussong et al., 2017). Collectively, these studies highlight a crucial role of BRD4 in regulation of alternative splicing. More work is needed to address how BRD4 functions to integrate the processes of chromatin structure, transcription, and splicing to ensure proper regulation of gene expression.

\section{BROMODOMAIN AND EXTRA-TERMINAL DOMAIN PROTEINS IN DNA DAMAGE RESPONSE AND GENOME INTEGRITY}

In addition to transcriptional control, BRD4 also plays a role in DNA double-strand break (DSB) repair (Figure 4). Genomewide DNA breaks are associated with enhanced $\mathrm{H} 4$ acetylation, BRD4 recruitment, and stable establishment of the DNA repair complex, as well as formation of oncogenic gene rearrangements by a non-homologous end joining (NHEJ) pathway. In clinical tumor samples, BRD4 levels are negatively associated with outcomes after prostate cancer radiation therapy (Li et al., 2018). Similarly, BRD2 promotes DNA repair with a spatially restricted chromatin domain. Tip60/KAT5 generates histone H4 acetylation at DSBs, which recruits BRD2 to protect the acetylated chromatin from being deacetylated by histone deacetylases (HDACs) and allows acetylation to spread along chromatin. This process results in the creation of a spatially restricted domain/boundary in chromatin, likely facilitating the binding of repair protein 53BP1 to DSB sites for repair (GursoyYuzugullu et al., 2017).

$\mathrm{BRD} 2$ and BRD4 also promote normal DNA replication origin firing and progression, as they bind and recruit TICRR, a key protein required for initiation of DNA replication, to DNA replication origins in hyperacetylated euchromatin (Sansam et al., 2018). BET proteins also promote genome integrity through regulation of replication stress response signaling. In response to exogenous and endogenous replication stress, BRD4 promotes intra-S-phase replication checkpoint CHK1 activation. Therefore, BRD4 inhibition sensitizes cancer cells to various replication stress-inducing agents (Zhang et al., 2018). In addition, BRD4 maintains spatiotemporal coordination of transcription with replication, thus preventing transcription-replication conflict (TRC) and DNA damage checkpoint signaling in oncogenic cells (Edwards et al., 2020; Lam, 2020). As a result, BET-BD inhibition induces HEXIM1and RAD51-dependent conflicts between transcription and replication, causing replication stress through a rapid overall increase in RNA synthesis (Bowry et al., 2018). Finally, systematic bromodomain protein screens also identified BRD4mediated R-loop suppression pathways in genome integrity (Kim et al., 2019). R-loops are intermediate structures of transcription that accumulate when transcriptional elongation is blocked by BRD4 inhibitors. In a subset of cancer cells, BRD4 inhibition results in R-loop accumulation, leading to cell death caused by transcription-replication collisions and DNA double-strand breaks during the S-phase (Lam et al., 2020). These studies suggest targeting BET proteins as a new therapeutic strategy of cancer in that inhibition of BRD4 induces cell death of oncogenic cells through mechanisms such as transcription-replication conflict, R-loop accumulation, and DNA damage.

\section{STRATEGIES TOWARD SELECTIVE MODULATION OF BROMODOMAIN AND EXTRA-TERMINAL DOMAIN PROTEIN FUNCTIONS}

\section{Distinct Functions of Individual Bromodomain and Extra-Terminal Domain Proteins}

Small molecules that target BET bromodomains are being tested for various diseases but do not discern between BET proteins. Studies have highlighted context-specific, distinct, and often opposing functions of BRD2, BRD3, BRD4, and BRDT. For example, distinct roles of BET proteins in ERa enhancer 
function and gene regulation have been demonstrated in breast cancer cells. While BRD2, BRD3, and BRD4 have partially redundant roles at $\mathrm{ERa}$ enhancers and gene transcription, a more unique role of $\mathrm{BRD} 3$ in $\mathrm{ERa}^{+}$breast cancer is revealed. BRD3 is recruited to a subset of ER-binding sites (ERBSs) enriched with active enhancer features in clusters likely functioning as super-enhancers and associated with highly estradiol (E2)-responsive genes (Murakami et al., 2019). In triple-negative breast cancer (TNBC), BRD2 positively regulates epithelial-to-mesenchymal transition (EMT), whereas BRD3 and BRD4 functionally repress this program with different transcriptional programs (Andrieu and Denis, 2018). Similarly, BRD4 is required for myogenic differentiation, whereas BRD3 represses the differentiation program (Roberts, 2017). In primary pancreatic stellate cells (PSCs) isolated from human pancreatic ductal adenocarcinoma (PDAC) tumors, BRD4 positively regulates collagen I expression in PSCs, while BRD2 and BRD3 negatively regulate it (Kumar, 2017). Importantly, the distinct functions of BRD4 and BRD2 could coordinate mouse ESC (mESC) control of pluripotency and exit that initiate lineage specification. BRD4 interacts with BRD9 in a bromodomaindependent fashion, leading to the recruitment of GBAFGLTSCR1 to form a smaller, non-canonical BAF complex, to coregulate the expression of key regulators of naïve mESC pluripotency (Gatchalian, 2018). BRD2 and BRD4 occupy Nodal gene regulatory elements (NREs), and BRD4 downregulation facilitates exit and drives enhanced BRD2 NRE occupancy to promote differentiative Nodal-Smad2 signaling (Fernandez-Alonso et al., 2017). Finally, the aberrant expression of BRDT in esophageal carcinoma (Wang et al., 2021b), ovarian cancer (Chen, 2020), and renal cell carcinoma (Wan et al., 2020) also contributes to the context-dependent function of BET proteins and is an attractive target for selective inhibition, in particular for different cancer types.

The molecular basis for differences in BET protein function within a given lineage has remained elusive. A recent study has performed an interactome profiling of BRD2, BRD3, BRD4, and BRDT interacting proteins and has gained significant insights into their selective interactions. As expected, the authors confirmed some shared interactors across all BET proteins. Notably, they noted that the p-TEFb and NELF complex is commonly associated with BRDT and BRD4, components of negative transcriptional regulators such as the NURD complex are associated with BRD3 and BRD4, while RNA Pol-II subunits and Mediator subunits are only identified as preferentially associated with BRD4. These data highlight the unique but versatile function of BRD4, suggesting an intricate interplay among BET proteins (Lambert et al., 2019). Finally, BRD2 and BRD3 have a similar domain structure and function to BRD4. An early study using fluorescence resonance energy transfer (FRET) to determine the interaction between cyan fluorescent protein (CFP)-tagged BRD2 and yellow fluorescent protein (YFP)-tagged histones provided important insights into the specificity of recognition of acetylated histones by BRD2. Specifically, BRD2 interacts with histone $\mathrm{H} 4 \mathrm{~K} 5 / \mathrm{K} 12$ but not $\mathrm{K} 8 / \mathrm{K} 16$, and to lesser degree H2B, but not other histones (Kanno et al., 2004). Studies highlighted the overlapping and non-overlapping functions of
$\mathrm{BRD} 2 / 3$ and BRD4 in gene transcription. In a defined in vitro chromatin transcription system, both BRD2 and BRD3 allowed RNA Pol-II to transcribe through nucleosomes depending on specific histone $\mathrm{H} 4$ modifications such as H4K5/12 (LeRoy et al., 2008). Interestingly, apart from acetylated histone, selective BRD2 recruitment has shown to be mediated by a histone variant H2A.Z.2, a driver of malignant melanoma to promote melanoma cell survival (Vardabasso et al., 2015). Similarly, selective interaction of BRD2 with Stat3(K87ac) has also been reported in Th17 cells by our own study (Cheung et al., 2017a). The current knowledge has agreed that BRD2 also plays an important role in cooperating with $\mathrm{CTCF} /$ cohesin components to define transcriptional and architectural boundaries, organizing chromatin complex formation, while BRD4 performs its function of transcriptional elongation (Cheung et al., 2017a; Hsu et al., 2017). Collectively, these studies suggest the collaborative nature of BET proteins in directing gene transcription programs in chromatin.

Importantly, using a gene-knockout rescue system in a $\mathrm{BRD}^{-/-}$erythroblast cell line, a series of mutant and chimeric BET proteins are functionally evaluated (Werner et al., 2020). BET N-terminal halves bearing BDs have a marked difference in protein stability but do not account for specificity in BET protein function. The function specificity is indeed mostly determined by the C-terminal half, as illustrated by a chimeric BET protein that consists of BRD4S-N and BRD2-C and functions similarly to intact BRD2. Part of the function for BRD2-C terminal functional specificity can be attributed to the coiled-coil (CC) domain, which functions in concert with the adjacent ET domain to impart BRD2-like activity onto BRD4S (Werner et al., 2020). These studies have collectively indicated that BET proteins in most cases are distinct in functions, which can be due to the intrinsic difference in C-terminal domains, leading to different interactomes that impart distinct functions in various contexts.

\section{TRANSCRIPTIONAL REPRESSOR FUNCTIONS OF BROMODOMAIN AND EXTRA-TERMINAL DOMAIN PROTEINS}

BRD4 inhibition leads to gene activation indirectly or directly, in contrast to the widely accepted positive role of BRD4 in gene expression. As an indirect mechanism, TIP60/BRD4 represses activation of endogenous retroviral elements and Irf7-mediated inflammatory response through positively regulating the expression of SUV39H1 and SETDB1 and global histone H3K9me3 in colon cancer (Rajagopalan et al., 2018). On the contrary, genes that are bounded by and repressed directly by BRD4 can also have functional significance. For example, BRD4 binds to the promoters of oxidative phosphorylation (OXPHOS) genes to repress the transcription. Upon BET-BD inhibitor treatment, BRD4 is displaced and OXPHOS gene expression increases which corrects bioenergetic deficiency caused by mitochondrial disease complex I mutations (Barrow et al., 2016). The mechanisms of how BRD4 represses gene transcription directly are currently under investigation. The silencing effect of a short isoform of BRD4S as a corepressor 
has been demonstrated in $\mathrm{HIV}-1$ transcription, through direct interaction of BRD4S with BRG1, a catalytic unit of BAF with known repressive function in $\mathrm{HIV}-1$ transcription, via its bromodomain and ET domain (Conrad et al., 2017). BRD4 can also interact with the methyltransferase G9a to repress a transcriptional program by di-methylating $\mathrm{H} 3 \mathrm{~K} 9$ that promotes genes involved in autophagy and lysosome biogenesis. BRD4 inhibition or, in the case of starvation of cells, repressive function of BRD4 is relieved, leading to autophagy gene activation and cell survival (Sakamaki et al., 2017; Sakamaki and Ryan, 2017). However, the interaction of BRD4/G9a can only partly explain the repression effect, underlying the need to further identify and characterize BRD4-repressive complexes. Interestingly, RING1B, a subunit of PRC1, colocalizes enhancers containing ERa in $\mathrm{ER}^{+}$ cells and to BRD4 enhancers in TNBC cells in active genes. Physical interaction of PRC1 and Fs(1)h and Br140, the Drosophila orthologs of BRD4 and BRD1, has also been confimed (Kang et al., 2017; Chan, 2018), indicating the BRD4-PRC1 repressive complex can play a functional role in some contexts yet to be determined. The transcriptionally repressive function of BRDT has also been demonstrated during spermatogenesis. BRDT undergoes complexation with HDAC1, PRMT5, and TRIM28 to transcriptionally repress the testis-specific histone H1t gene (Wang and Wolgemuth, 2016). Taken together, while previous studies demonstrated the dominant role of BET proteins in transcriptional activation, these studies have added another layer of complexity by showing that the context-dependent BET-interacting repressive complex can repress gene transcription with functional significance. Therefore, it will be necessary to evaluate the therapeutic efficacies of strategies selectively targeting the BET-repressive complex in the future.

\section{BRD4 SHORT ISOFORM VS LONG ISOFORM}

The expression levels of BRD isoforms BRD4L and BRD4S seem to be determined by the function of splicing kinase SRPK1, which influences BRD4 occupancy at genomic loci of myc and bcl2 in leukemogenesis (Tzelepis, 2018). There are currently three identified BRD4 isoforms, and their functional distinctions are beginning to be unraveled, pointing to the opposing role of BRD4S vs. BRD4L forms. In human cancer cells, BRD4S forms nuclear puncta that possess liquid-like properties and colocalizes with BRD4L, MED1, and histone H3K27Ac. BRD4 puncta correlate with BRD4S but not the BRD4L expression level. BRD4S plays a substantially larger role than BRD4L in incorporating BRD4 condensations in chromatin and is facilitated by its tandem BDs binding to lysine-acetylated histones (Han et al., 2020). Function of BRD4S has been demonstrated in the context of human papillomaviruses (HPVs) that encode E2 protein to recruit viral and cellular proteins. BRD4 is a highly conserved interactor with E2 protein. BRD4S lacks the CTM but has an intact phosphordependent interaction domain (PDID) and basic interaction domain (BID) and can interact with HPV31 E2 through the
PDID and repress E2 activities, in contrast to the activator function of BRD4L on E2-mediated viral gene transcription (Yigitliler, 2021). Opposing functions of BRD4 isoforms have also been studied in breast cancer, showing the oncogenic EN1-BRD4S axis and tumor-suppressive BRD4L in breast cancer, which demonstrates the context-dependent functions of long and short isoforms (Wu et al., 2020). As aforementioned, the function of BET proteins is mostly determined by the C-terminal half and the associated interactome that dictates their functions in specificity contexts; it is therefore important to characterize the functional distinctions of BRD4L vs. BRD4S in different disease contexts.

\section{TOXICITY OF BROMODOMAIN AND EXTRA-TERMINAL DOMAIN PROTEIN INHIBITION IN VIVO}

BET-BD inhibitors have shown therapeutic efficacies in preclinical models. However, the role of BRD4 in the normal cell developmental process and subsequently sustained BET-BD inhibition in normal tissue toxicity has been reported in clinical evaluation of various BET-BD inhibitors but not well characterized. Analysis of Brd4-KO mice using the $\mathrm{Brd} 4^{\mathrm{fl} / \mathrm{fl}}$ system has shown that BRD4 is required for hematopoietic stem cell expansion and progenitor development but has a limited role in macrophage development (Dey, 2019). On the contrary, using an inducible and reversible transgenic RNAi mouse model, Lowe and colleagues showed that sustained suppression of BRD4 in adult mice displayed reversible epidermal hyperplasia, alopecia, and decreased cellular diversity and stem cell depletion in the small intestine (Bolden et al., 2014). These studies therefore highlight the ubiquitous functions of BRD4 in different tissues. They also suggest that although BET-BD inhibition can be developed as a therapeutic approach for inflammatory diseases due to the reversibility of toxicity, treatment protocols need to be optimized to achieve therapeutic efficacy while avoiding prolonged suppression to allow for tissue recovery.

\section{SELECTIVE FUNCTION AND TARGETING OF BROMODOMAIN AND EXTRA-TERMINAL DOMAIN BD1 VS. BD2 IN DRUG DEVELOPMENT}

To minimize the toxicity of pan-BET protein inhibition in vivo, recent studies have focused on developing strategies that selectively target individual BET proteins or individual bromodomains. Importantly, this strategy has also been demonstrated with beneficial effects in cancer treatment. BET-BD1 and BET-BD2 selective inhibitors have been shown to be better therapeutic strategies for cancer or inflammatory diseases, respectively (Gilan et al., 2020), as toxicity associated with single BET-BD inhibition appears to be significantly lower than that of pan-BET-BD inhibition (Faivre et al., 2020). However, the dichotomous usage of BET-BD1 and -BD2 inhibitors in cancer and inflammatory diseases needs to be further 
refined with more investigation of their detailed mechanism of action. Cheung et al. reported that BRD4-BD1 inhibition selectively blocks T-helper (Th)17 cell differentiation and ameliorates colitis (Cheung et al., 2017b). Our unpublished results also have revealed that selective targeting of BRD4-BD2 inhibits Th2 cell differentiation, thereby paving the way for the applications of BRD4 BD1- vs. BD2selective inhibitors to selectively target Th17- or Th2-related immune diseases.

\section{CONCLUSION}

The major advances in bromodomain biology in the recent years have greatly enhanced our mechanistic understanding of the diverse and versatile functions of BET proteins in the complex regulation of gene transcription in chromatin in biology as well as human diseases. It has become increasingly evident that BET proteins exert distinct and overlapping functions collectively with many other key transcription and chromatin regulatory proteins that enable cells to execute gene transcription programs in response to physiological and pathophysiological cues. It is therefore necessary to characterize and understand the

\section{REFERENCES}

Anand, P., Brown, J. D., Lin, C. Y., Qi, J., Zhang, R., Artero, P. C., et al. (2013). BET Bromodomains Mediate Transcriptional Pause Release in Heart Failure. Cell 154, 569-582. doi:10.1016/j.cell.2013.07.013

Andrieu, G. P., and Denis, G. V. (2018). BET Proteins Exhibit Transcriptional and Functional Opposition in the Epithelial-To-Mesenchymal Transition. Mol. Cancer Res. 16, 580-586. doi:10.1158/1541-7786.mcr-17-0568

Asangani, I. A., Dommeti, V. L., Wang, X., Malik, R., Cieslik, M., Yang, R., et al. (2014). Therapeutic Targeting of BET Bromodomain Proteins in CastrationResistant Prostate Cancer. Nature. 510, 278-282. doi:10.1038/nature13229

Au-Yeung, N., and HorvathHistone, C. M. H2A. (2018). Histone H2A.Z Suppression of Interferon-Stimulated Transcription and Antiviral Immunity Is Modulated by GCN5 and BRD2. iScience. 6, 68-82. doi:10.1016/jisci.2018.07.013

Barrow, J. J., Balsa, E., Verdeguer, F., Tavares, C. D. J., Soustek, M. S., Hollingsworth, L. R., et al. (2016). Bromodomain Inhibitors Correct Bioenergetic Deficiency Caused by Mitochondrial Disease Complex I Mutations. Mol. Cel. 64, 163-175. doi:10.1016/j.molcel.2016.08.023

Bhattacharyya, S., Pradhan, K., Campbell, N., Mazdo, J., Vasantkumar, A., Maqbool, S., et al. (2017). Altered Hydroxymethylation Is Seen at Regulatory Regions in Pancreatic Cancer and Regulates Oncogenic Pathways. Genome Res. 27, 1830-1842. doi:10.1101/gr.222794.117

Bolden, J. E., Tasdemir, N., Dow, L. E., van Es, J. H., Wilkinson, J. E., Zhao, Z., et al. (2014). Inducible In Vivo Silencing of Brd4 Identifies Potential Toxicities of Sustained BET Protein Inhibition. Cel. Rep. 8, 1919-1929. doi:10.1016/ j.celrep.2014.08.025

Bowry, A., Piberger, A. L., Rojas, P., Saponaro, M., and Petermann, E. (2018). BET Inhibition Induces HEXIM1- and RAD51-dependent Conflicts between Transcription and Replication. Cel. Rep. 25, 2061-2069. doi:10.1016/ j.celrep.2018.10.079e2064

Chan, H. L. (2018). Polycomb Complexes Associate with Enhancers and Promote Oncogenic Transcriptional Programs in Cancer through Multiple Mechanisms. Nat. Commun. 9, 3377. doi:10.1038/s41467-018-05728-x

Chen, L. (2020). BRDT Promotes Ovarian Cancer Cell Growth. Cell Death Dis. 11, 1021. doi:10.1038/s41419-020-03225-y

Cheung, K., Lu, G., Sharma, R., Vincek, A., Zhang, R., Plotnikov, A. N., et al. (2017). BET N-Terminal Bromodomain Inhibition Selectively Blocks Th17 Cell Differentiation and Ameliorates Colitis in Mice. Proc. Natl. Acad. Sci. USA. 114, 2952-2957. doi:10.1073/pnas.1615601114 context-specific functions of BET proteins for the purpose of developing novel small molecule bromodomain inhibitors that are capable of selectively modulating BET protein functions to control gene transcription in a given functional setting. Such bromodomain inhibitors likely have great potential as new epigenetic therapies for a wide array of human diseases including cancer, inflammatory and autoimmune diseases, and cardiovascular disorders.

\section{AUTHOR CONTRIBUTIONS}

All authors listed have made a substantial, direct, and intellectual contribution to the work and approved it for publication.

\section{FUNDING}

This work was supported in part by the research grants from Crohn's \& Colitis Foundation (KLC; Award\# 579304) and the National Institutes of Health (M-MZ; R01AI124465, R01CA239165, R01AG072562).

Cheung, K. L., Zhang, F., Jaganathan, A., Sharma, R., Zhang, Q., Konuma, T., et al. (2017). Distinct Roles of Brd2 and Brd4 in Potentiating the Transcriptional Program for Th17 Cell Differentiation. Mol. Cel. 65, 1068-1080. doi:10.1016/ j.molcel.2016.12.022

ChiangPhospho-Brd4, C.-M. (2016). Phospho-BRD4: Transcription Plasticity and Drug Targeting. Drug Discov. Today Tech. 19, 17-22. doi:10.1016/ j.ddtec.2016.05.003

Choe, J., Lin, S., Zhang, W., Liu, Q., Wang, L., Ramirez-Moya, J., et al. (2018). mRNA Circularization by METTL3-eIF3h Enhances Translation and Promotes Oncogenesis. Nature. 561, 556-560. doi:10.1038/s41586-018-0538-8

Conrad, R. J., Fozouni, P., Thomas, S., Sy, H., Zhang, Q., Zhou, M.-M., et al. (2017). The Short Isoform of BRD4 Promotes HIV-1 Latency by Engaging Repressive SWI/SNF Chromatin-Remodeling Complexes. Mol. Cel. 67, 1001-1012. doi:10.1016/j.molcel.2017.07.025

Crump, N. T. (2021). BET Inhibition Disrupts Transcription but Retains EnhancerPromoter Contact. Nat. Commun. 12, 223. doi:10.1038/s41467-020-20400-Z

Dai, X., Gan, W., Li, X., Wang, S., Zhang, W., Huang, L., et al. (2017). Prostate Cancer-Associated SPOP Mutations Confer Resistance to BET Inhibitors through Stabilization of BRD4. Nat. Med. 23, 1063-1071. doi:10.1038/ $\mathrm{nm} .4378$

Daneshvar, K., Ardehali, M. B., Klein, I. A., Hsieh, F.-K., Kratkiewicz, A. J., Mahpour, A., et al. (2020). IncRNA DIGIT and BRD3 Protein Form PhaseSeparated Condensates to Regulate Endoderm Differentiation. Nat. Cel Biol. 22, 1211-1222. doi:10.1038/s41556-020-0572-2

Dawson, M. A., Prinjha, R. K., Dittmann, A., Giotopoulos, G., Bantscheff, M., Chan, W. I., et al. (2011). Inhibition of BET Recruitment to Chromatin as an Effective Treatment for MLL-Fusion Leukaemia. Nature. 478, 529-533. doi:10.1038/nature 10509

Delmore, J. E., Issa, G. C., Lemieux, M. E., Rahl, P. B., Shi, J., Jacobs, H. M., et al. (2011). BET Bromodomain Inhibition as a Therapeutic Strategy to Target C-Myc. Cell. 146, 904-917. doi:10.1016/j.cell.2011.08.017

Devaiah, B. N., Case-Borden, C., Gegonne, A., Hsu, C. H., Chen, Q., Meerzaman, D., et al. (2016). BRD4 Is a Histone Acetyltransferase that Evicts Nucleosomes from Chromatin. Nat. Struct. Mol. Biol. 23, 540-548. doi:10.1038/nsmb.3228

Devaiah, B. N., Mu, J., Akman, B., Uppal, S., Weissman, J. D., Cheng, D., et al. (2020). MYC Protein Stability Is Negatively Regulated by BRD4. Proc. Natl. Acad. Sci. USA. 117, 13457-13467. doi:10.1073/pnas.1919507117

Dey, A. (2019). BRD4 Directs Hematopoietic Stem Cell Development and Modulates Macrophage Inflammatory Responses. EMBO J. 38. doi:10.15252/ embj.2018100293 
Dhalluin, C., Carlson, J. E., Zeng, L., He, C., Aggarwal, A. K., Zhou, M.-M., et al. (1999). Structure and Ligand of a Histone Acetyltransferase Bromodomain. Nature. 399, 491-496. doi:10.1038/20974

Dhar, S., Thota, A., and Rao, M. R. S. (2012). Insights into Role of Bromodomain, Testis-specific (Brdt) in Acetylated Histone H4-dependent Chromatin Remodeling in Mammalian Spermiogenesis. J. Biol. Chem. 287, 6387-6405. doi:10.1074/jbc.m111.288167

Di Micco, R., Fontanals-Cirera, B., Low, V., Ntziachristos, P., Yuen, S. K., Lovell, C. D., et al. (2014). Control of Embryonic Stem Cell Identity by BRD4-dependent Transcriptional Elongation of Super-enhancer-associated Pluripotency Genes. Cell Rep. 9, 234-247. doi:10.1016/j.celrep.2014.08.055

Edwards, D. S., Maganti, R., Tanksley, J. P., Luo, J., Park, J. J. H., Balkanska-Sinclair, E., et al. (2020). BRD4 Prevents R-Loop Formation and TranscriptionReplication Conflicts by Ensuring Efficient Transcription Elongation. Cel. Rep. 32, 108166. doi:10.1016/j.celrep.2020.108166

Erber, L., Luo, A., and Chen, Y. (2019). Targeted and Interactome Proteomics Revealed the Role of PHD2 in Regulating BRD4 Proline Hydroxylation. Mol. Cell Proteomics. 18, 1772-1781. doi:10.1074/mcp.ra119.001535

Faivre, E. J., McDaniel, K. F., Albert, D. H., Mantena, S. R., Plotnik, J. P., Wilcox, D., et al. (2020). Selective Inhibition of the BD2 Bromodomain of BET Proteins in Prostate Cancer. Nature. 578, 306-310. doi:10.1038/s41586-020-1930-8

Fernandez-Alonso, R., Davidson, L., Hukelmann, J., Zengerle, M., Prescott, A. R., Lamond, A., et al. (2017). Brd4-Brd2 Isoform Switching Coordinates Pluripotent Exit and Smad2-dependent Lineage Specification. EMBO Rep. 18, 1108-1122. doi:10.15252/embr.201643534

Filippakopoulos, P., Picaud, S., Mangos, M., Keates, T., Lambert, J.-P., BarsyteLovejoy, D., et al. (2012). Histone Recognition and Large-Scale Structural Analysis of the Human Bromodomain Family. Cell 149, 214-231. doi:10.1016/j.cell.2012.02.013

Filippakopoulos, P., Qi, J., Picaud, S., Shen, Y., Smith, W. B., Fedorov, O., et al. (2010). Selective Inhibition of BET Bromodomains. Nature. 468, 1067-1073. doi:10.1038/nature09504

Flynn, E. M., Huang, O. W., Poy, F., Oppikofer, M., Bellon, S. F., Tang, Y., et al. (2015). A Subset of Human Bromodomains Recognizes Butyryllysine and Crotonyllysine Histone Peptide Modifications. Structure. 23, 1801-1814. doi:10.1016/j.str.2015.08.004

Fontanals-Cirera, B., Hasson, D., Vardabasso, C., Di Micco, R., Agrawal, P., Chowdhury, A., et al. (2017). Harnessing BET Inhibitor Sensitivity Reveals AMIGO2 as a Melanoma Survival Gene. Mol. Cel. 68, 731-744. doi:10.1016/ j.molcel.2017.11.004e739

French, C. A., Rahman, S., Walsh, E. M., Kühnle, S., Grayson, A. R., Lemieux, M. E., et al. (2014). NSD3-NUT Fusion Oncoprotein in NUT Midline Carcinoma: Implications for a Novel Oncogenic Mechanism. Cancer Discov. 4, 928-941. doi:10.1158/2159-8290.cd-14-0014

Gatchalian, J. (2018). A Non-canonical BRD9-Containing BAF Chromatin Remodeling Complex Regulates Naive Pluripotency in Mouse Embryonic Stem Cells. Nat. Commun. 9, 5139. doi:10.1038/s41467-018-07528-9

Gibson, B. A., Doolittle, L. K., Schneider, M. W. G., Jensen, L. E., Gamarra, N., Henry, L., et al. (2019). Organization of Chromatin by Intrinsic and Regulated Phase Separation. Cell. 179, 470-484. doi:10.1016/j.cell.2019.08.037

Gilan, O., Rioja, I., Knezevic, K., Bell, M. J., Yeung, M. M., Harker, N. R., et al. (2020). Selective Targeting of BD1 and BD2 of the BET Proteins in Cancer and Immunoinflammation. Science. 368, 387-394. doi:10.1126/science.aaz8455

Goudarzi, A., Zhang, D., Huang, H., Barral, S., Kwon, O. K., Qi, S., et al. (2016). Dynamic Competing Histone H4 K5K8 Acetylation and Butyrylation Are Hallmarks of Highly Active Gene Promoters. Mol. Cel. 62, 169-180. doi:10.1016/j.molcel.2016.03.014

Guo, W. (2020). Role of BRD4 Phosphorylation in the Nucleus Accumbens in Relapse to Cocaine-Seeking Behavior in Mice. Addict. Biol. 25, e12808. doi:10.1111/adb.12808

Gursoy-Yuzugullu, O., Carman, C., and Price, B. D. (2017). Spatially Restricted Loading of BRD2 at DNA Double-Strand Breaks Protects H4 Acetylation Domains and Promotes DNA Repair. Sci. Rep. 7, 12921. doi:10.1038/s41598017-13036-5

Han, X., Yu, D., Gu, R., Jia, Y., Wang, Q., Jaganathan, A., et al. (2020). Roles of the BRD4 Short Isoform in Phase Separation and Active Gene Transcription. Nat. Struct. Mol. Biol. 27, 333-341. doi:10.1038/s41594-020-0394-8
Hargreaves, D. C., Horng, T., and Medzhitov, R. (2009). Control of Inducible Gene Expression by Signal-dependent Transcriptional Elongation. Cell. 138, 129-145. doi:10.1016/j.cell.2009.05.047

Hsu, S. C., Gilgenast, T. G., Bartman, C. R., Edwards, C. R., Stonestrom, A. J., Huang, P., et al. (2017). The BET Protein BRD2 Cooperates with CTCF to Enforce Transcriptional and Architectural Boundaries. Mol. Cel. 66, 102-116. doi:10.1016/j.molcel.2017.02.027

Hu, X., Dong, S.-H., Chen, J., Zhou, X. Z., Chen, R., Nair, S., et al. (2017). Prolyl Isomerase PIN1 Regulates the Stability, Transcriptional Activity and Oncogenic Potential of BRD4. Oncogene. 36, 5177-5188. doi:10.1038/ onc. 2017.137

Hussong, M., Kaehler, C., Kerick, M., Grimm, C., Franz, A., Timmermann, B., et al. (2017). The Bromodomain Protein BRD4 Regulates Splicing during Heat Shock. Nucleic Acids Res. 45, 382-394. doi:10.1093/nar/gkw729

Jang, M. K., Mochizuki, K., Zhou, M., Jeong, H.-S., Brady, J. N., and Ozato, K. (2005). The Bromodomain Protein Brd4 Is a Positive Regulatory Component of P-TEFb and Stimulates RNA Polymerase II-dependent Transcription. Mol. Cel. 19, 523-534. doi:10.1016/j.molcel.2005.06.027

Janouskova, H., El Tekle, G., Bellini, E., Udeshi, N. D., Rinaldi, A., Ulbricht, A. et al. (2017). Opposing Effects of Cancer-type-specific SPOP Mutants on BET Protein Degradation and Sensitivity to BET Inhibitors. Nat. Med. 23, 1046-1054. doi:10.1038/nm.4372

Jin, X., Yan, Y., Wang, D., Ding, D., Ma, T., Ye, Z., et al. (2018). DUB3 Promotes BET Inhibitor Resistance and Cancer Progression by Deubiquitinating BRD4. Mol. Cel. 71, 592-605. doi:10.1016/j.molcel.2018.06.036

Jonkers, I., and Lis, J. T. (2015). Getting up to Speed with Transcription Elongation by RNA Polymerase II. Nat. Rev. Mol. Cel Biol. 16, 167-177. doi:10.1038/nrm3953

Kang, H., Jung, Y. L., McElroy, K. A., Zee, B. M., Wallace, H. A., Woolnough, J. L., et al. (2017). Bivalent Complexes of PRC1 with Orthologs of BRD4 and MOZ/ MORF Target Developmental Genes inDrosophila. Genes Dev. 31, 1988-2002. doi:10.1101/gad.305987.117

Kanno, T., Kanno, Y., LeRoy, G., Campos, E., Sun, H.-W., Brooks, S. R., et al. (2014). BRD4 Assists Elongation of Both Coding and Enhancer RNAs by Interacting with Acetylated Histones. Nat. Struct. Mol. Biol. 21, 1047-1057. doi: $10.1038 / \mathrm{nsmb} .2912$

Kanno, T., Kanno, Y., Siegel, R. M., Jang, M. K., Lenardo, M. J., and Ozato, K. (2004). Selective Recognition of Acetylated Histones by Bromodomain Proteins Visualized in Living Cells. Mol. Cel. 13, 33-43. doi:10.1016/s1097-2765(03) $00482-9$

Kim, C. R., Noda, T., Kim, H., Kim, G., Park, S., Na, Y., et al. (2020). PHF7 Modulates BRDT Stability and Histone-To-Protamine Exchange during Spermiogenesis. Cel Rep. 32, 107950. doi:10.1016/j.celrep.2020.107950

Kim, J. J., Lee, S. Y., Gong, F., Battenhouse, A. M., Boutz, D. R., Bashyal, A., et al. (2019). Systematic Bromodomain Protein Screens Identify Homologous Recombination and R-Loop Suppression Pathways Involved in Genome Integrity. Genes Dev. 33, 1751-1774. doi:10.1101/gad.331231.119

Kohnken, R., Wen, J., Mundy-Bosse, B., McConnell, K., Keiter, A., Grinshpun, L., et al. (2018). Diminished microRNA-29b Level Is Associated with BRD4Mediated Activation of Oncogenes in Cutaneous T-Cell Lymphoma. Blood. 131, 771-781. doi:10.1182/blood-2017-09-805663

Konuma, T. (2017). Structural Mechanism of the Oxygenase JMJD6 Recognition by the Extraterminal (ET) Domain of BRD4. Sci. Rep. 7, 16272. doi:10.1038/ s41598-017-16588-8

Korb, E., Herre, M., Zucker-Scharff, I., Darnell, R. B., and Allis, C. D. (2015). BET Protein Brd4 Activates Transcription in Neurons and BET Inhibitor Jq1 Blocks Memory in Mice. Nat. Neurosci. 18, 1464-1473. doi:10.1038/nn.4095

Krainer, G. (2021). Reentrant Liquid Condensate Phase of Proteins Is Stabilized by Hydrophobic and Non-ionic Interactions. Nat. Commun. 12, 1085. doi:10.1038/s41467-021-21181-9

Krishna, V., Yin, X., Song, Q., Walsh, A., Pocalyko, D., Bachman, K., et al. (2021). Integration of the Transcriptome and Genome-wide Landscape of BRD2 and BRD4 Binding Motifs Identifies Key Superenhancer Genes and Reveals the Mechanism of Bet Inhibitor Action in Rheumatoid Arthritis Synovial Fibroblasts. J.I. 206, 422-431. doi:10.4049/jimmunol.2000286

Kumar, K. (2017). BET Inhibitors Block Pancreatic Stellate Cell Collagen I Production and Attenuate Fibrosis In Vivo. JCI Insight. 2, e88032. doi:10.1172/jci.insight. 88032 
Lam, F. C. (2020). BRD4 Prevents the Accumulation of R-Loops and Protects against Transcription-Replication Collision Events and DNA Damage. Nat. Commun. 11, 4083. doi:10.1038/s41467-020-17503-y

Lam, F. C., Kong, Y. W., and Yaffe, M. B. (2020). Inducing DNA Damage through R-Loops to Kill Cancer Cells. Mol. Cell Oncol. 8, 1848233. doi:10.1080/ 23723556.2020.1848233

Lambert, J.-P., Picaud, S., Fujisawa, T., Hou, H., Savitsky, P., Uusküla-Reimand, L., et al. (2019). Interactome Rewiring Following Pharmacological Targeting of BET Bromodomains. Mol. Cel. 73, 621-638. doi:10.1016/j.molcel.2018.11.006

Lee, S. (2020). JMJD6 Cleaves MePCE to Release Positive Transcription Elongation Factor B (P-TEFb) in Higher Eukaryotes. Elife. 9. doi:10.7554/elife.53930

LeRoy, G., Rickards, B., and Flint, S. J. (2008). The Double Bromodomain Proteins Brd2 and Brd3 Couple Histone Acetylation to Transcription. Mol. Cel. 30, 51-60. doi:10.1016/j.molcel.2008.01.018

Li, X., Baek, G., Ramanand, S. G., Sharp, A., Gao, Y., Yuan, W., et al. (2018). BRD4 Promotes DNA Repair and Mediates the Formation of TMPRSS2-ERG Gene Rearrangements in Prostate Cancer. Cel. Rep. 22, 796-808. doi:10.1016/ j.celrep.2017.12.078

Li, Y., Sabari, B. R., Panchenko, T., Wen, H., Zhao, D., Guan, H., et al. (2016). Molecular Coupling of Histone Crotonylation and Active Transcription by AF9 YEATS Domain. Mol. Cel. 62, 181-193. doi:10.1016/j.molcel.2016.03.028

Liu, W., Ma, Q., Wong, K., Li, W., Ohgi, K., Zhang, J., et al. (2013). Brd4 and JMJD6-Associated Anti-pause Enhancers in Regulation of Transcriptional Pause Release. Cell. 155, 1581-1595. doi:10.1016/j.cell.2013.10.056

Lovén, J., Hoke, H. A., Lin, C. Y., Lau, A., Orlando, D. A., Vakoc, C. R., et al. (2013). Selective Inhibition of Tumor Oncogenes by Disruption of Super-enhancers. Cell. 153, 320-334. doi:10.1016/j.cell.2013.03.036

Lu, Y., Wu, T., Gutman, O., Lu, H., Zhou, Q., Henis, Y. I., et al. (2020). Phase Separation of TAZ Compartmentalizes the Transcription Machinery to Promote Gene Expression. Nat. Cel. Biol. 22, 453-464. doi:10.1038/s41556020-0485-0

Ma, L. (2021). Co-condensation between Transcription Factor and Coactivator P300 Modulates Transcriptional Bursting Kinetics. Mol. Cel. 81, 1682-1697. doi:10.1016/j.molcel.2021.01.031

Martin, R. D. (2020). Differential Activation of P-TEFb Complexes in the Development of Cardiomyocyte Hypertrophy Following Activation of Distinct G Protein-Coupled Receptors. Mol. Cel Biol. 40. doi:10.1128/ mcb.00048-20

Miller, T. C., Simon, B., RybinGrötsch, V. H., Curtet, S., Khochbin, S., Carlomagno, T., et al. (2016). A Bromodomain-DNA Interaction Facilitates Acetylationdependent Bivalent Nucleosome Recognition by the BET Protein BRDT. Nat. Commun. 7, 13855. doi:10.1038/ncomms13855

Morinière, J., Rousseaux, S., Steuerwald, U., Soler-López, M., Curtet, S., Vitte, A.-L., et al. (2009). Cooperative Binding of Two Acetylation marks on a Histone Tail by a Single Bromodomain. Nature. 461, 664-668. doi:10.1038/nature08397

Murakami, S., Li, R., Nagari, A., Chae, M., Camacho, C. V., and Kraus, W. L. (2019). Distinct Roles for BET Family Members in Estrogen Receptor a Enhancer Function and Gene Regulation in Breast Cancer Cells. Mol. Cancer Res. 17, 2356-2368. doi:10.1158/1541-7786.mcr-19-0393

Nakamura, Y., Umehara, T., Nakano, K., Jang, M. K., Shirouzu, M., Morita, S., et al. (2007). Crystal Structure of the Human BRD2 Bromodomain: Insights into Dimerization and Recognition of Acetylated Histone H4. J. Biol. Chem. 282, 4193-4201. doi:10.1074/jbc.M605971200

Nicodeme, E., Jeffrey, K. L., Schaefer, U., Beinke, S., Dewell, S., Chung, C.-w., et al. (2010). Suppression of Inflammation by a Synthetic Histone Mimic. Nature. 468, 1119-1123. doi:10.1038/nature09589

Patel, K., Solomon, P. D., Walshe, J. L., Ford, D. J., Wilkinson-White, L., Payne, R. J., et al. (2021). BET-family Bromodomains Can Recognize Diacetylated Sequences from Transcription Factors Using a Conserved Mechanism. Biochemistry. 60, 648-662. doi:10.1021/acs.biochem.0c00816

Patel, K., Solomon, P. D., Walshe, J. L., Low, J. K. K., and Mackay, J. P. (2021). The Bromodomains of BET Family Proteins Can Recognize Diacetylated Histone H2A .Z. Protein Sci. 30, 464-476. doi:10.1002/pro.4006

Pistoni, M. (2021). Long Noncoding RNA NEAT1 Acts as a Molecular Switch for BRD4 Transcriptional Activity and Mediates Repression of BRD4/WDR5 Target Genes. Mol. Cancer Res. 19, 799-811. doi:10.1158/1541-7786.mcr-20-0324

Qin, Z. Y., Wang, T., Su, S., Shen, L. T., Zhu, G. X., Liu, Q., et al. (2019). BRD4 Promotes Gastric Cancer Progression and Metastasis through Acetylation- dependent Stabilization of Snail. Cancer Res. 79, 4869-4881. doi:10.1158/00085472.CAN-19-0442

Rahman, S., Sowa, M. E., Ottinger, M., Smith, J. A., Shi, Y., Harper, J. W., et al. (2011). The Brd4 Extraterminal Domain Confers Transcription Activation Independent of pTEFb by Recruiting Multiple Proteins, Including NSD3. Mol. Cel. Biol. 31, 2641-2652. doi:10.1128/mcb.01341-10

Rahnamoun, H., Lee, J., Sun, Z., Lu, H., Ramsey, K. M., Komives, E. A., et al. (2018). RNAs Interact with BRD4 to Promote Enhanced Chromatin Engagement and Transcription Activation. Nat. Struct. Mol. Biol. 25, 687-697. doi:10.1038/ s41594-018-0102-0

Rajagopalan, D., Tirado-Magallanes, R., Bhatia, S. S., Teo, W. S., Sian, S., Hora, S., et al. (2018). TIP60 Represses Activation of Endogenous Retroviral Elements. Nucleic Acids Res. 46, 9456-9470. doi:10.1093/nar/gky659

Reynoird, N., Schwartz, B. E., Delvecchio, M., Sadoul, K., Meyers, D., Mukherjee, C., et al. (2010). Oncogenesis by Sequestration of CBP/p300 in Transcriptionally Inactive Hyperacetylated Chromatin Domains. EMBO J. 29, 2943-2952. doi:10.1038/emboj.2010.176

Roberts, T. C. (2017). BRD3 and BRD4 BET Bromodomain Proteins Differentially Regulate Skeletal Myogenesis. Sci. Rep. 7, 6153. doi:10.1038/s41598-017-06483-7

Roe, J.-S., Mercan, F., Rivera, K., Pappin, D. J., and Vakoc, C. R. (2015). BET Bromodomain Inhibition Suppresses the Function of Hematopoietic Transcription Factors in Acute Myeloid Leukemia. Mol. Cel. 58, 1028-1039. doi:10.1016/j.molcel.2015.04.011

Sabari, B. R. (2018). Coactivator Condensation at Super-enhancers Links Phase Separation and Gene Control. Science. 361. doi:10.1126/science.aar3958

Sakamaki, J.-I., and Ryan, K. M. (2017). Transcriptional Regulation of Autophagy and Lysosomal Function by Bromodomain Protein BRD4. Autophagy. 13, 2006-2007. doi:10.1080/15548627.2017.1364822

Sakamaki, J.-i., Wilkinson, S., Hahn, M., Tasdemir, N., O’Prey, J., Clark, W., et al. (2017). Bromodomain Protein BRD4 Is a Transcriptional Repressor of Autophagy and Lysosomal Function. Mol. Cel. 66, 517-532. doi:10.1016/j.molcel.2017.04.027

Sansam, C. G., Pietrzak, K., Majchrzycka, B., Kerlin, M. A., Chen, J., Rankin, S., et al. (2018). A Mechanism for Epigenetic Control of DNA Replication. Genes Dev. 32, 224-229. doi:10.1101/gad.306464.117

Schröder, S., Cho, S., Zeng, L., Zhang, Q., Kaehlcke, K., Mak, L., et al. (2012). Twopronged Binding with Bromodomain-Containing Protein 4 Liberates Positive Transcription Elongation Factor B from Inactive Ribonucleoprotein Complexes. J. Biol. Chem. 287, 1090-1099. doi:10.1074/jbc.m111.282855

Shang, E., Wang, X., Wen, D., Greenberg, D. A., and Wolgemuth, D. J. (2009). Double Bromodomain-Containing Gene Brd2 Is Essential for Embryonic Development in Mouse. Dev. Dyn. 238, 908-917. doi:10.1002/dvdy.21911

Shen, C., Ipsaro, J. J., Shi, J., Milazzo, J. P., Wang, E., Roe, J.-S., et al. (2015). NSD3Short Is an Adaptor Protein that Couples BRD4 to the CHD8 Chromatin Remodeler. Mol. Cel. 60, 847-859. doi:10.1016/j.molcel.2015.10.033

Shi, J., Wang, Y., Zeng, L., Wu, Y., Deng, J., Zhang, Q., et al. (2014). Disrupting the Interaction of BRD4 with Diacetylated Twist Suppresses Tumorigenesis in Basal-like Breast Cancer. Cancer Cell. 25, 210-225. doi:10.1016/ j.ccr.2014.01.028

Shi, W., Zhang, C., Ning, Z., Hua, Y., Li, Y., Chen, L., et al. (2019). Long Noncoding RNA LINC00346 Promotes Pancreatic Cancer Growth and Gemcitabine Resistance by Sponging miR-188-3p to Derepress BRD4 Expression. J. Exp. Clin. Cancer Res. 38, 60. doi:10.1186/s13046-019$1055-9$

Shu, S., Lin, C. Y., He, H. H., Witwicki, R. M., Tabassum, D. P., Roberts, J. M., et al. (2016). Response and Resistance to BET Bromodomain Inhibitors in TripleNegative Breast Cancer. Nature. 529, 413-417. doi:10.1038/nature16508

Soffers, J. H. M., Li, X., Abmayr, S. M., and Workman, J. L. (2016). Reading and Interpreting the Histone Acylation Code. Genomics, Proteomics of Bioinformatics. 14, 329-332. doi:10.1016/j.gpb.2016.12.001

Stratton, M. S., Lin, C. Y., Anand, P., Tatman, P. D., Ferguson, B. S., Wickers, S. T., et al. (2016). Signal-Dependent Recruitment of BRD4 to Cardiomyocyte Super-enhancers Is Suppressed by a MicroRNA. Cel Rep. 16, 1366-1378. doi:10.1016/j.celrep.2016.06.074

Suzuki, H. I., Young, R. A., and Sharp, P. A. (2017). Super-Enhancer-Mediated RNA Processing Revealed by Integrative MicroRNA Network Analysis. Cell. 168, 1000-1014. doi:10.1016/j.cell.2017.02.015

Szczepanski, A. P. (2020). ASXL3 Bridges BRD4 to BAP1 Complex and Governs Enhancer Activity in Small Cell Lung Cancer. Genome Med. 12, 63. doi:10.1186/ s13073-020-00760-3 
Tang, K., Zhao, J., Xie, J., and Wang, J. (2019). Decreased miR-29b Expression Is Associated with Airway Inflammation in Chronic Obstructive Pulmonary Disease. Am. J. Physiology-Lung Cell Mol. Physiol. 316, L621-L629. doi:10.1152/ajplung.00436.2018

Tasdemir, N., Banito, A., Roe, J.-S., Alonso-Curbelo, D., Camiolo, M., Tschaharganeh, D. F., et al. (2016). BRD4 Connects Enhancer Remodeling to Senescence Immune Surveillance. Cancer Discov. 6, 612-629. doi:10.1158/2159-8290.cd-16-0217

Tonouchi, E., Gen, Y., Muramatsu, T., Hiramoto, H., Tanimoto, K., Inoue, J., et al. (2018). miR-3140 Suppresses Tumor Cell Growth by Targeting BRD4 via its Coding Sequence and Downregulates the BRD4-NUT Fusion Oncoprotein. Sci. Rep. 8, 4482. doi:10.1038/s41598-018-24861-7

Tzelepis, K. (2018). SRPK1 Maintains Acute Myeloid Leukemia through Effects on Isoform Usage of Epigenetic Regulators Including BRD4. Nat. Commun. 9, 5378. doi:10.1038/s41467-018-07620-0

Uppal, S., Gegonne, A., Chen, Q., Thompson, P. S., Cheng, D., Mu, J., et al. (2019). The Bromodomain Protein 4 Contributes to the Regulation of Alternative Splicing. Cel Rep. 29, 2450-2460. doi:10.1016/j.celrep.2019.10.066

Vardabasso, C., Gaspar-Maia, A., Hasson, D., Pünzeler, S., Valle-Garcia, D., Straub, T., et al. (2015). Histone Variant H2A.Z.2 Mediates Proliferation and Drug Sensitivity of Malignant Melanoma. Mol. Cel. 59, 75-88. doi:10.1016/ j.molcel.2015.05.009

Wai, D. C. C., Szyszka, T. N., Campbell, A. E., Kwong, C., Wilkinson-White, L. E., Silva, A. P. G., et al. (2018). The BRD3 ET Domain Recognizes a Short Peptide Motif through a Mechanism that Is Conserved across Chromatin Remodelers and Transcriptional Regulators. J. Biol. Chem. 293, 7160-7175. doi:10.1074/jbc.ra117.000678

Wan, P., Chen, Z., Zhong, W., Jiang, H., Huang, Z., Peng, D., et al. (2020). BRDT Is a Novel Regulator of eIF4EBP1 in Renal Cell Carcinoma. Oncol. Rep. 44, 2475-2486. doi:10.3892/or.2020.7796

Wang, F., Liu, H., Blanton, W. P., Belkina, A., Lebrasseur, N. K., and Denis, G. V. (2009). Brd2 Disruption in Mice Causes Severe Obesity without Type 2 Diabetes. Biochem. J. 425, 71-85. doi:10.1042/bj20090928

Wang, L. T., Liu, K-Y., Jeng, W-Y., Chiang, C-M., Chai, C-Y., Chiou, S-S., et al. (2020). PCAF-mediated Acetylation of ISX Recruits BRD4 to Promote Epithelial-Mesenchymal Transition. EMBO Rep. 21, e48795. doi:10.15252/ embr.201948795

Wang, L., and Wolgemuth, D. J. (2016). BET Protein BRDT Complexes with HDAC1, PRMT5, and TRIM28 and Functions in Transcriptional Repression during Spermatogenesis. J. Cel. Biochem. 117, 1429-1438. doi:10.1002/jcb.25433

Wang, N., Wu, R., Tang, D., and Kang, R. (2021). The BET Family in Immunity and Disease. Signal. Transduct Target. Ther. 6, 23. doi:10.1038/s41392-020-00384-4

Wang, R., Cao, X.-J., Kulej, K., Liu, W., Ma, T., MacDonald, M., et al. (2017). Uncovering BRD4 Hyperphosphorylation Associated with Cellular Transformation in NUT Midline Carcinoma. Proc. Natl. Acad. Sci. USA. 114, E5352-E5361. doi:10.1073/pnas.1703071114

Wang, R., Li, Q., Helfer, C. M., Jiao, J., and You, J. (2012). Bromodomain Protein Brd4 Associated with Acetylated Chromatin Is Important for Maintenance of Higher-Order Chromatin Structure. J. Biol. Chem. 287, 10738-10752. doi:10.1074/jbc.m111.323493

Wang, X., Kutschat, A. P., Yamada, M., Prokakis, E., Böttcher, P., Tanaka, K., et al. (2021). Bromodomain Protein BRDT Directs $\triangle$ Np63 Function and Superenhancer Activity in a Subset of Esophageal Squamous Cell Carcinomas. Cell Death Differ. 28, 2207-2220. doi:10.1038/s41418-021-00751-w

Welti, J., Sharp, A., Yuan, W., Dolling, D., Nava Rodrigues, D., Figueiredo, I., et al. (2018). Targeting Bromodomain and Extra-terminal (BET) Family Proteins in Castration-Resistant Prostate Cancer (CRPC). Clin. Cancer Res. 24, 3149-3162. doi:10.1158/1078-0432.ccr-17-3571

Werner, M. T., Wang, H., Hamagami, N., Hsu, S. C., Yano, J. A., Stonestrom, A. J., et al. (2020). Comparative Structure-Function Analysis of Bromodomain and Extraterminal Motif (BET) Proteins in a Gene-Complementation System. J. Biol. Chem. 295, 1898-1914. doi:10.1074/jbc.ra119.010679

Wong, M. (2019). JMJD6 Is a Tumorigenic Factor and Therapeutic Target in Neuroblastoma. Nat. Commun. 10, 3319. doi:10.1038/s41467-019-11132-w

Wu, S.-Y., and Chiang, C.-M. (2007). The Double Bromodomain-Containing Chromatin Adaptor Brd4 and Transcriptional Regulation. J. Biol. Chem. 282, 13141-13145. doi:10.1074/jbc.r700001200

Wu, S.-Y., Lee, A.-Y., Lai, H.-T., Zhang, H., and Chiang, C.-M. (2013). Phospho Switch Triggers Brd4 Chromatin Binding and Activator Recruitment for Genespecific Targeting. Mol. Cel. 49, 843-857. doi:10.1016/j.molcel.2012.12.006
Wu, S.-Y., Lee, C.-F., Lai, H.-T., Yu, C.-T., Lee, J.-E., Zuo, H., et al. (2020). Opposing Functions of BRD4 Isoforms in Breast Cancer. Mol. Cel. 78, 1114-1132. doi:10.1016/j.molcel.2020.04.034

Wu, T., Kamikawa, Y. F., and Donohoe, M. E. (2018). Brd4's Bromodomains Mediate Histone H3 Acetylation and Chromatin Remodeling in Pluripotent Cells through P300 and Brg1. Cel Rep. 25, 1756-1771. doi:10.1016/ j.celrep.2018.10.003

Xie, F., Xiao, X., Tao, D., Huang, C., Wang, L., Liu, F., et al. (2020). circNR3C1 Suppresses Bladder Cancer Progression through Acting as an Endogenous Blocker of BRD4/C-Myc Complex. Mol. Ther. - Nucleic Acids. 22, 510-519. doi:10.1016/j.omtn.2020.09.016

Xu, S., Fan, L., Jeon, H.-Y., Zhang, F., Cui, X., Mickle, M. B., et al. (2020). p300-Mediated Acetylation of Histone Demethylase JMJD1A Prevents its Degradation by Ubiquitin Ligase STUB1 and Enhances its Activity in Prostate Cancer. Cancer Res. 80, 3074-3087. doi:10.1158/0008-5472.can-20-0233

Yang, Z., Yik, J. H. N., Chen, R., He, N., Jang, M. K., Ozato, K., et al. (2005). Recruitment of $\mathrm{P}-\mathrm{TEFb}$ for Stimulation of Transcriptional Elongation by the Bromodomain Protein Brd4. Mol. Cel. 19, 535-545. doi:10.1016/ j.molcel.2005.06.029

Yigitliler, A. (2021). BRD4S Interacts with Viral E2 Protein to Limit Human Papillomavirus Late Transcription. J. Virol. 95, e02032-20. doi:10.1128/ jvi.02032-20

Zaware, N., and Zhou, M.-M. (2019). Bromodomain Biology and Drug Discovery. Nat. Struct. Mol. Biol. 26, 870-879. doi:10.1038/s41594-019-0309-8

Zhang, J., Dulak, A. M., Hattersley, M. M., Willis, B. S., Nikkilä, J., Wang, A., et al. (2018). BRD4 Facilitates Replication Stress-Induced DNA Damage Response. Oncogene. 37, 3763-3777. doi:10.1038/s41388-018-0194-3

Zhang, P., Wang, D., Zhao, Y., Ren, S., Gao, K., Ye, Z., et al. (2017). Intrinsic BET Inhibitor Resistance in SPOP-Mutated Prostate Cancer Is Mediated by BET Protein Stabilization and AKT-mTORC1 Activation. Nat. Med. 23, 1055-1062. doi: $10.1038 / \mathrm{nm} .4379$

Zhang, Q., Zeng, L., Shen, C., Ju, Y., Konuma, T., Zhao, C., et al. (2016). Structural Mechanism of Transcriptional Regulator NSD3 Recognition by the ET Domain of BRD4. Structure. 24, 1201-1208. doi:10.1016/j.str.2016.04.019

Zheng, B., Aoi, Y., Shah, A. P., Iwanaszko, M., Das, S., Rendleman, E. J., et al. (2021). Acute Perturbation Strategies in Interrogating RNA Polymerase II Elongation Factor Function in Gene Expression. Genes Dev. 35, 273-285. doi:10.1101/gad.346106.120

Zheng, G., Schweiger, M.-R., Martinez-Noel, G., Zheng, L., Smith, J. A., Harper, J. W., et al. (2009). Brd4 Regulation of Papillomavirus Protein E2 Stability. J. Virol. 83, 8683-8692. doi:10.1128/jvi.00674-09

Zippo, A., Serafini, R., Rocchigiani, M., Pennacchini, S., Krepelova, A., and Oliviero, S. (2009). Histone Crosstalk between H3S10ph and H4K16ac Generates a Histone Code that Mediates Transcription Elongation. Cell. 138, 1122-1136. doi:10.1016/j.cell.2009.07.031

Zuber, J., Shi, J., Wang, E., Rappaport, A. R., Herrmann, H., Sison, E. A., et al. (2011). RNAi Screen Identifies Brd4 as a Therapeutic Target in Acute Myeloid Leukaemia. Nature. 478, 524-528. doi:10.1038/nature10334

Conflict of Interest: M-MZ is a founder, director, and shareholder of Parkside Scientific, Inc.

The remaining authors declare that the research was conducted in the absence of any commercial or financial relationships that could be construed as a potential conflict of interest.

Publisher's Note: All claims expressed in this article are solely those of the authors and do not necessarily represent those of their affiliated organizations, or those of the publisher, the editors, and the reviewers. Any product that may be evaluated in this article, or claim that may be made by its manufacturer, is not guaranteed or endorsed by the publisher.

Copyright $\odot 2021$ Cheung, Kim and Zhou. This is an open-access article distributed under the terms of the Creative Commons Attribution License (CC BY). The use, distribution or reproduction in other forums is permitted, provided the original author(s) and the copyright owner(s) are credited and that the original publication in this journal is cited, in accordance with accepted academic practice. No use, distribution or reproduction is permitted which does not comply with these terms. 